\title{
Polar cap ion beams during periods of northward IMF: Cluster statistical results
}

\author{
R. Maggiolo ${ }^{1}$, M. Echim ${ }^{1,2}$, J. De Keyser ${ }^{1}$, D. Fontaine ${ }^{3}$, C. Jacquey ${ }^{4}$, and I. Dandouras ${ }^{4}$ \\ ${ }^{1}$ Belgian Institute for Space Aeronomy, Space Plasma, Brussels, Belgium \\ ${ }^{2}$ Institute for Space Sciences, Bucharest, Romania \\ ${ }^{3}$ Laboratoire de Physique des Plasmas, Velizy, France \\ ${ }^{4}$ Institut de Recherche en Astrophysique et Planétologie, UPS-CNRS, Toulouse, France
}

Received: 14 January 2011 - Revised: 11 March 2011 - Accepted: 7 April 2011 - Published: 6 May 2011

\begin{abstract}
Above the polar caps and during prolonged periods of northward IMF, the Cluster satellites detect upward accelerated ion beams with energies up to a few keV. They are associated with converging electric field structures indicating that the acceleration is caused by a quasi-static fieldaligned electric field that can extend to altitudes higher than $7 R_{\mathrm{E}}$ (Maggiolo et al., 2006; Teste et al., 2007).

Using the AMDA science analysis service provided by the Centre de Données de la Physique des Plasmas, we have been able to extract about 200 events of accelerated upgoing ion beams above the polar caps from the Cluster database. Most of these observations are taken at altitudes lower than $7 R_{\mathrm{E}}$ and in the Northern Hemisphere.
\end{abstract}

We investigate the statistical properties of these ion beams. We analyze their geometry, the properties of the plasma populations and of the electric field inside and around the beams, as well as their dependence on solar wind and IMF conditions. We show that $\sim 40 \%$ of the ion beams are collocated with a relatively hot and isotropic plasma population. The density and temperature of the isotropic population are highly variable but suggest that this plasma originates from the plasma sheet. The ion beam properties do not change significantly when the isotropic, hot background population is present. Furthermore, during one single polar cap crossing by Cluster it is possible to detect upgoing ion beams both with and without an accompanying isotropic component.

The analysis of the variation of the IMF $B_{\mathrm{Z}}$ component prior to the detection of the beams indicates that the delay between a northward/southward turning of IMF and the appearance/disappearance of the beams is respectively $\sim 2 \mathrm{~h}$ and $20 \mathrm{~min}$. The observed electrodynamic characteristics of high altitude polar cap ion beams suggest that they are closely connected to polar cap auroral arcs. We discuss the impli-

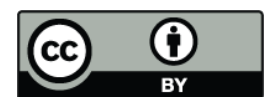

Correspondence to: R. Maggiolo (romain.maggiolo@aeronomie.be) cations of these Cluster observations above the polar cap on the magnetospheric dynamics and configuration during prolonged periods of northward IMF.

Keywords. Magnetospheric physics (Auroral phenomena; Magnetosphere-ionosphere interactions; Polar cap phenomena)

\section{Introduction}

Field-aligned acceleration by quasi-static electric fields has been widely reported in the auroral zone (e.g. Mozer et al., 1977; Reiff et al., 1988; Ergun et al., 1998). Various signatures are clearly associated with this process. The presence of an accelerating electric field along magnetic field lines manifests itself by strong electric fields perpendicular to the magnetic field at high altitude (Mozer et al., 1977). Electric field equipotentials exhibit a U- or S-shaped profile and the field-aligned electric field is located at relatively low altitudes, typically below $2.5 R_{\mathrm{E}}$ (Mozer and Hull, 2001). This electric field accelerates ionospheric ions upward and magnetospheric electrons downward, creating a current system that couples the local ionosphere with more distant magnetospheric regions. The U- or S-shaped potential drop produces the well known inverted-V structure in particle spectrograms, respectively for precipitating electrons at low altitude and for outflowing ions at high altitudes. An analytical quasi-stationary analysis has been recently proposed by De Keyser et al. (2010). The downward quasi-static acceleration of electrons excites optical emissions in the ionosphere and leads to the formation of elongated and relatively stable discrete auroral arcs (e.g. Kletzing et al., 1983).

Partamies et al. (2008) showed that the inverted-V structures associated with precipitating accelerated electrons are mainly located at auroral latitudes $\left(65^{\circ}-75^{\circ}\right)$ and that their statistical spatial distribution and size are actually similar to

Published by Copernicus Publications on behalf of the European Geosciences Union. 
those of discrete auroral arcs as observed from the ground. Partamies et al. (2008) also reported detection of inverted$\mathrm{V}$ structures at invariant latitudes higher than $80^{\circ}$. Fieldaligned acceleration by parallel electric fields at high invariant latitude, above the polar caps, has been reported since the 1980s (see the review by Zhu et al., 1997, for a detailed historic account).

Evidence for electron inverted-V spectra associated with optical emissions and discrete arcs above the polar caps was given by low altitude spacecraft like S3-2 (Burke et al., 1982). Frank et al. (1986) showed similar observations by the DE spacecraft for theta aurora. Optical emissions in the polar caps are a typical feature of the northward IMF polar caps. They consist of discrete and elongated optical structures that are generally oriented in the sun-earth direction and have relatively long lifetimes. Several endeavors have been made to classify the polar cap arcs depending on their location, geometry, and shape or connection to the auroral oval (see the review by Zhu et al., 1997). While they have been widely studied, mainly from optical data and low altitude spacecraft data, the physical processes that generate polar cap arcs are still unclear.

At high altitude and above the polar caps, outflowing ion beams with inverted- $\mathrm{V}$ structures have been observed by Cluster at altitudes ranging from 4 to $8 R_{\mathrm{E}}$ during prolonged periods of northward IMF (Maggiolo et al. 2006). These ion beams are locally accelerated by quasi-static electric field structures with a field-aligned electric field extending to higher altitude (up to $5 R_{\mathrm{E}}$ ) than the parallel E-field of the auroral zone. Teste et al. (2007) showed that the upgoing field-aligned ion beams detected above the polar caps are surrounded by upgoing electron beams. The upgoing ion and electron beams form a succession of upward and downward current sheets of similar intensity, suggesting a local closure of this current system. A detailed examination of one particular event showed that the ion outflow structure was elongated in the $X_{\mathrm{GSE}}$ direction and had a lifetime of more than $30 \mathrm{~min}$.

The purpose of this paper is to study the statistical properties of the high latitude upgoing ion beams measured by Cluster above the polar caps. One main goal of this study is to compare the statistical properties of polar cap ion beams (PCIB) to that of polar cap arcs and of inverted-V structures observed in the auroral zone. The paper is organized as follows: Sect. 2 describes the data and the method developed to select the ion events; Sect. 3 presents a statistical analysis of the ion properties inside the beams, as well as of beam geometry and size. Sections 4 and 5 discuss the dependence on IMF and solar wind plasma properties and the location of PCIB. In the last section, we summarize the results and discuss them in the context of the magnetospheric configuration during prolonged periods of northward IMF.

\section{Instrumentation and event selection}

The four identical Cluster satellites were launched in 2001 on an elliptical orbit $\left(4.0 \times 19.6 R_{\mathrm{E}}\right)$ with an inclination of $90^{\circ}$. A detailed description of the Cluster mission can be found in Escoubet et al. (2001).

Ion data are provided by the Cluster Ion Spectrometers (CIS) onboard the Cluster spacecraft 1, 3 and 4 (Rème et al., 2001).

In addition, we use electric field data from EDI (Paschmann et al., 2001) and/or EFW experiments (Gustafsson et al., 1997). Note that EFW computes the two spin plane components of the electric field (Dawn-Dusk and SunEarth) from the tip-to-tip electric potential difference between probes at the ends of two booms deployed on the Cluster spacecraft. The third component of the electric field is computed by assuming that the electric field along the magnetic field direction is null. Magnetic field data come from the Cluster Fluxgate Magnetometers (FGM) (Balogh et al., 2001).

We define a PCIB as a region where the ion bulk velocity is strongly aligned with the magnetic field, surrounded by lobe plasma and accelerated by a quasi-static electric field. The typical signature of quasi-static acceleration is the presence of a convergent electric field with a potential drop across the beam comparable to the upgoing ion energy. All these characteristics have been integrated into a procedure for semiautomatic detection of events. The event selection proceeds in two steps.

(1) An automatic detection of upgoing ion beams inside the lobes using AMDA, a tool developed by the CDPP (the French national data center for natural plasmas of the solar system), and (2) a user-controlled cross-check of "bad" events, including the verification that the ion beam acceleration is consistent with a quasi-static electric field. Let us detail the two steps below.

AMDA makes a conditional search on satellite data and gives as an output the time periods when mathematical conditions specified by the user are satisfied. In our study we used a combination of conditions on the ion moments determined by the CIS/HIA detector onboard Cluster. The method and results for each condition are illustrated in Fig. 1.

The first condition (A) was to detect field-aligned ion beams. We browsed CIS/HIA ion data to find time periods when (A.1) the ion velocity along the magnetic field was higher than $70 \mathrm{~km} \mathrm{~s}^{-1}$, and (A.2) the ion density was higher than $0.03 \mathrm{~cm}^{-3}$. The ion density threshold was defined in order to remove low density regions (typically inside the lobes) where counting rates are too low to give an accurate estimate of the ion velocity.

The lobe region is populated by cold plasma undetected by the HIA experiment, as it is not energetic enough to overcome the positive spacecraft potential and reach the CIS detector. Thus, to automatically detect the lobe region in the HIA dataset, we used a criterion on density. This second 


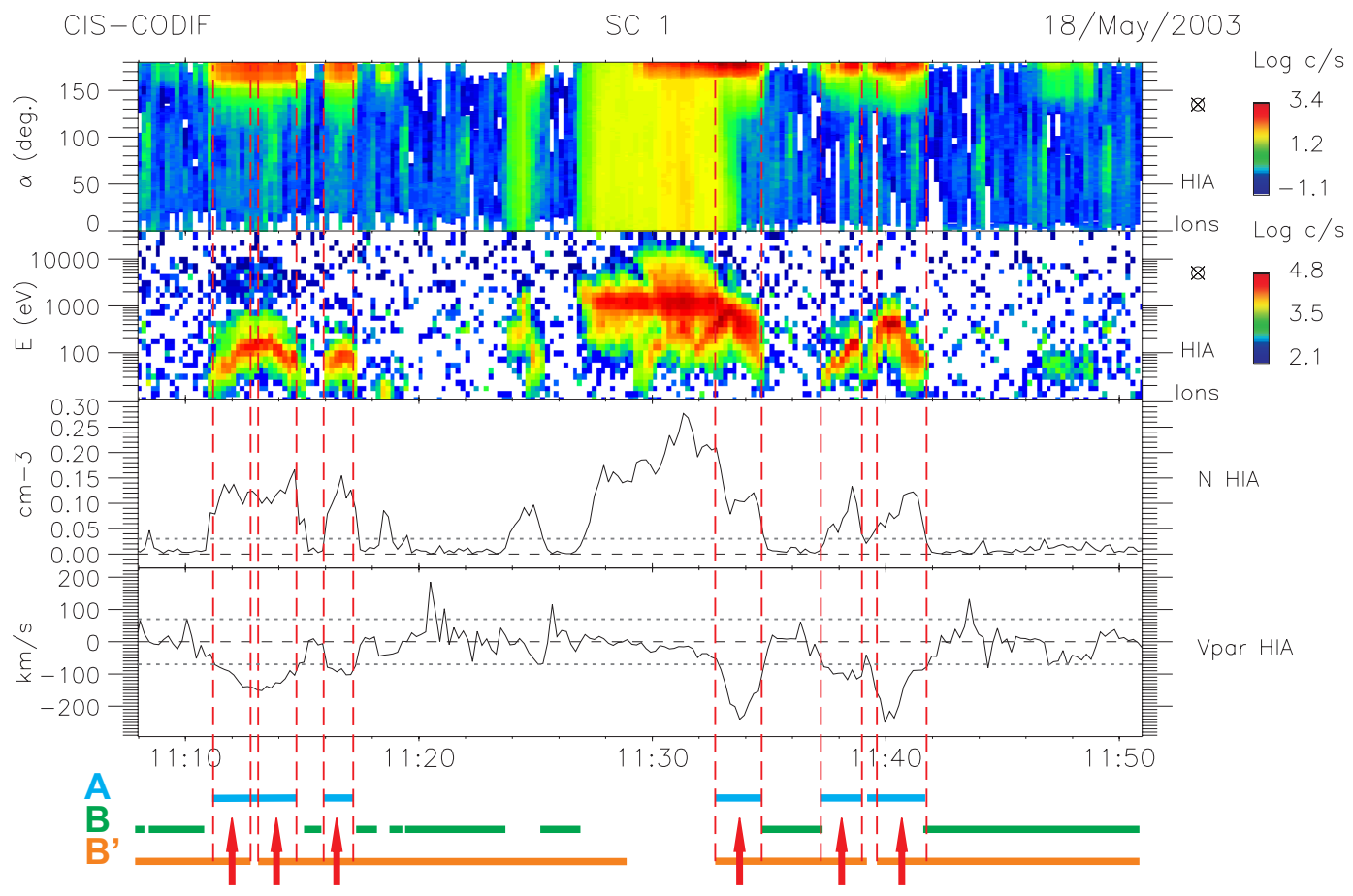

Fig. 1. Data from Cluster 1 on 18 May 2003. From top to bottom: ion pitch angle distribution from HIA for all ions; time-energy spectrogram from HIA for all ions; ion density computed from HIA; ion parallel velocity computed from HIA. The horizontal colored lines at the bottom of the figure represent the time periods when condition A $\left(n>0.03 \mathrm{~cm}^{-3}\right.$ and $\left.V_{\mathrm{par}}>70 \mathrm{~km} \mathrm{~s}^{-1}\right)$ is satisfied (light blue) and when condition B $\left(n<0.03 \mathrm{~cm}^{-3}\right)$ is satisfied (green). The orange horizontal line represents the condition $\mathrm{B}^{\prime}$, which is the extension ( \pm 2 min) of condition $\mathrm{B}$. The time periods delimited by vertical red dashed lines and indicated by red arrows correspond to the intersection between condition A and $\mathrm{B}^{\prime}$.

condition (B) is satisfied when the measured plasma density is lower than $0.03 \mathrm{~cm}^{-3}$. The size of the region where this condition is satisfied may depend on the IMF orientation favoring specific IMF orientation in our event selection procedure. Indeed, during periods of southward IMF, part of the lobe region may be filled by ionospheric ions outflowing from the cusp/cleft region and energetic enough to be detected by HIA (Maggiolo et al., 2006; Nilsson et al., 2006). On the other hand, during periods of northward IMF the lobe region shrinks (e.g. Makita et al., 1983). However, there is observational evidence that most of the time - and whatever is the IMF orientation - there exists a lobe region only populated by cold plasma undetected by particle detectors (e.g. Moore et al., 1997; Engwall et al., 2009). Thus, condition B can be satisfied for any IMF orientation.

Finally, to obtain the time periods when Cluster detects field-aligned ion beams inside the lobe region we combined these two conditions. Obviously, because of the density threshold, condition A and B exclude each other. We thus defined a new condition $\mathrm{B}^{\prime}$ by extending the time periods satisfying condition $\mathrm{B}$ by $\pm 2 \mathrm{~min}$.

The intersection between condition $\mathrm{A}$ and $\mathrm{B}^{\prime}$ correspond to periods when field-aligned ion beams are detected by Cluster in the magnetospheric lobes. Figure 1 shows CIS/HIA data when Cluster detects upward accelerated ion beams above the polar caps. This example illustrates how the conditions defined for the AMDA tool are able to automatically detect the upgoing ion beams above the polar cap. Note that no condition has been given on the Cluster orbit and that the regions of interest, i.e. the magnetospheric lobes, are selected by a criterion on particle density (condition $\mathrm{B}$ ).

Some of the events selected automatically do not correspond exactly to what is expected. First, ion beams located at the lobe-plasma sheet interface do satisfy our condition $\left(\mathrm{A}+\mathrm{B}^{\prime}\right)$; nevertheless they are observed at the poleward boundary of the auroral oval and are not surrounded by lobe plasma on both sides and have therefore been removed from our database.

We also removed events that satisfied our condition $\left(A+B^{\prime}\right)$ due to spurious values of the density and/or velocity, like is the case, for instance, at times when the CIS/HIA experiment changes its operating mode.

Outflowing ions originating from the cusp/cleft region are frequently observed in the lobes. They are accelerated at low altitude above the dayside polar ionosphere and are transported in the tailward direction by the convection electric field. As shown by Maggiolo et al. (2006), they have energy and temperature signatures similar to the PCIB and can also 


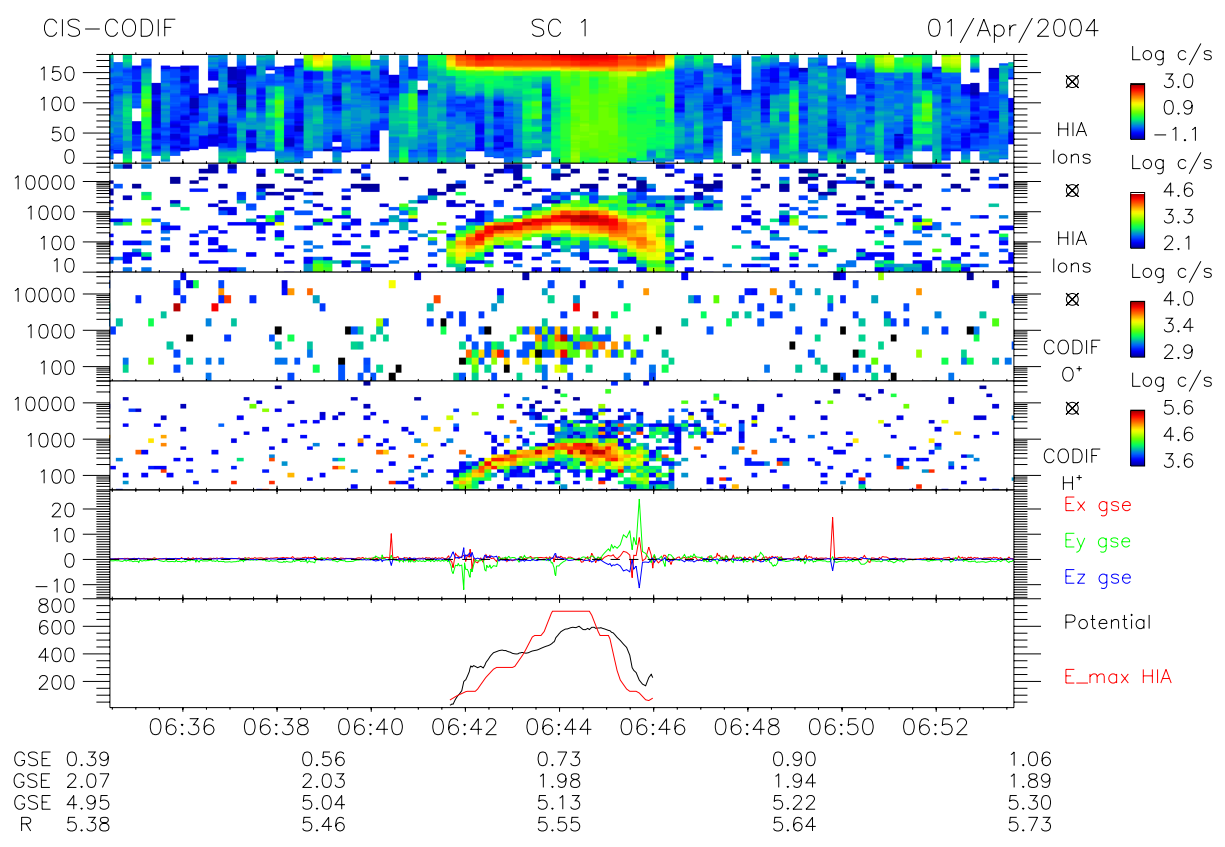

Fig. 2. Data from Cluster 1 on 1 April 2004. From top to bottom: ion pitch angle distribution from HIA for all ions; time-energy spectrogram from HIA for all ions; time-energy spectrogram from CODIF for $\mathrm{O}^{+}$; time-energy spectrogram from CODIF for $\mathrm{H}^{+}$; GSE components of the perpendicular electric field as given by EFW; electric potential along the spacecraft trajectory computed from EFW data (black curve) and energy of the maximum of ion fluxes (red curve). The polar cap ion beam is detected between 06:42 UT and 06:46 UT.

display energy variations resembling inverted V structures. Despite these similarities, cusp/cleft outflows can be distinguished from PCIB without any ambiguity. Indeed, while they move upward along the magnetic field lines, ions from the cusp/cleft drift at the ExB velocity perpendicularly to the magnetic field. The resulting velocity filter effect provides a mass dispersion. Consequently, at a given location above the polar caps, ions of different masses are well separated in energy. This is clearly observed by Cluster. Maggiolo et al. (2006) reported energy ratio between $\mathrm{O}^{+}$and $\mathrm{H}^{+}$around 4 at distances of 5-9 $R_{\mathrm{E}}$ and Nilsson et al. (2006) around 16 in the 5 to $14 R_{\mathrm{E}}$ distance region. This characteristic of cusp/cleft ion outflows rules out any confusion between them and PCIB. We removed cusp/cleft ion outflows from our database by checking that the ion field-aligned acceleration was electrostatic. As illustrated in Fig. 2, we verified that (i) $\mathrm{H}^{+}$and $\mathrm{O}^{+}$had the same energy, (ii) the ion beams are associated with strong perpendicular electric fields, and (iii) the electric potential integrated along the Cluster orbit is matching the ion beam energy.

The algorithm described above has been applied to Cluster 1 data from the beginning of 2001 to the end of 2006, and identified a set of 185 events. Our selection criteria on density, velocity, and electric field signature are somewhat arbitrary. Therefore, it is possible that the real number of events is larger. Note that only 15 events are located in the Southern Hemisphere. This is an effect of the Cluster orbit (see Sect. 5) and doesn't imply that PCIB are more frequent in the Northern Hemisphere. However, as $92 \%$ of the events of our dataset are located in the Northern Hemisphere, our results are only representative of PCIB located in this hemisphere.

\section{Properties of PCIB}

This section focuses on the properties of the ion populations detected inside the acceleration structures, on the orientation and size of the beams, and on the estimation of the total ion outflow associated with PCIB.

\subsection{Energy of locally accelerated PCIB}

The outflowing ions have temperatures of the order of tens of $\mathrm{eV}$ and contain a significant amount of $\mathrm{O}^{+}$ions. They are strongly field-aligned and move in the tailward direction along the magnetic field. We can therefore conjecture that their origin is the ionosphere.

The properties of the outflowing ions in the center of the beams - where the ion energy is maximal - are summarized in Fig. 3.

Figure 3a shows the distribution of the energy of the peak flux as given by the HIA experiment. The typical ion energy is below $500 \mathrm{eV}$ with an average energy of about $370 \mathrm{eV}$. However, there is a high energy tail in this distribution and some beams may reach energies up to $\sim 2 \mathrm{keV}$. Note that because of the condition on parallel velocity defined for the 

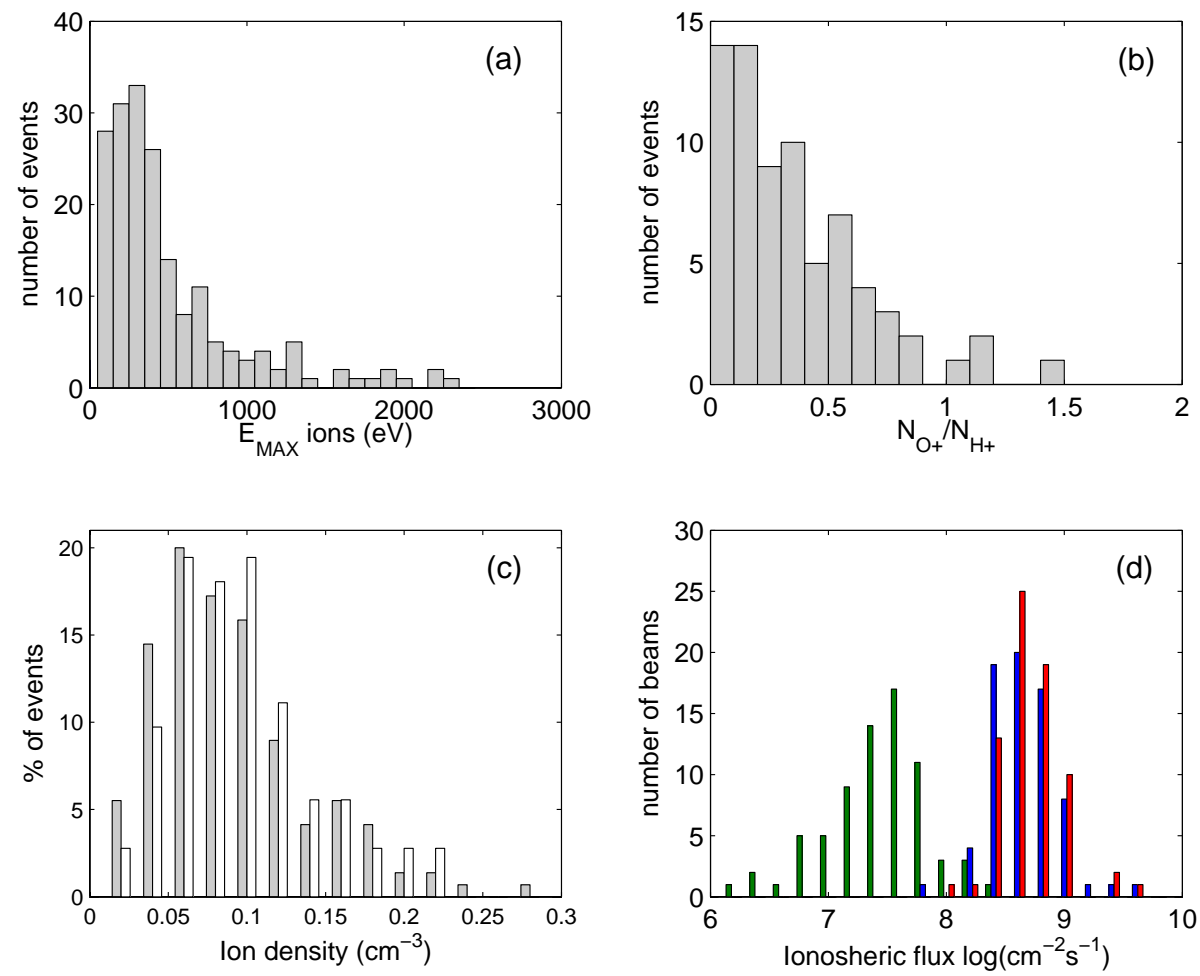

Fig. 3. Properties of PCIB. (a) Ion energy of the peak flux computed from HIA. (b) $\mathrm{O}^{+}$to $\mathrm{H}^{+}$density ratio computed from CODIF. (c) Outflowing ions density computed from HIA (grey bars) and from CODIF (white bars). There are less data points for CODIF than for HIA, as CODIF was off after April 2005. For better comparison the distributions have been normalized. The ion density computed from CODIF is the sum of the density of outflowing $\mathrm{H}^{+}$and $\mathrm{O}^{+}$. The contribution of other species is considered as negligible. (d) Particle flux mapped to ionospheric altitude $(100 \mathrm{~km})$. The green bars stand for $\mathrm{O}^{+}$and the blue bars for $\mathrm{H}^{+}$, and the red bars correspond to the total flux (obtained by summing $\mathrm{O}^{+}$and $\mathrm{H}^{+}$flux).

automatic detection of events, ion beams with energies below $25 \mathrm{eV}$ are excluded from this distribution.

The energy of the outflowing ion beams corresponds to the energy to which they have been accelerated along the magnetic field and thus to the field-aligned potential drop between the ionosphere and the Cluster spacecraft. The satellite altitude may influence the observed peak energy when the spacecraft is located inside the acceleration region. Consequently the acceleration that occurs above Cluster can be missed. Indeed, the study by Maggiolo et al. (2006) revealed that the field-aligned electric field can extend above Cluster altitude. However, for the events presented in that study, at least 75 percent of the total field aligned potential drop was located below Cluster altitude. Furthermore, we found no correlation between Cluster altitude and the peak energy in our dataset. So we conclude that the ion peak energy is a good proxy of the total field-aligned potential drop and that the bias due to Cluster altitude in the energy distribution is low.

In contrast to the auroral zone, where the total fieldaligned potential drop ranges in most cases from 2 to $4 \mathrm{keV}$ and can reach $\sim 15 \mathrm{keV}$ (e.g. Partamies et al., 2008), above the polar caps, it is almost one order of magnitude lower.
However, in both regions the distribution of the field-aligned potential displays similar profiles with more events at low energies and a tail at high energies with a decreasing exponential profile (with a characteristic energy of $\sim 400 \mathrm{eV}$ for PCIB).

\subsection{Density and composition of PCIB}

Figure $3 b$, c, and d, respectively, shows the distribution of the $\mathrm{O}^{+}$to $\mathrm{H}^{+}$number density ratio from CODIF, the total ion density obtained from CIS/HIA and CIS/CODIF, and the total ion flux. Note that the CODIF detector onboard Cluster 1 was off after April 2005 so that there are less data points for ion composition and CODIF density than the number of events in our dataset.

The total density obtained from HIA, which is computed assuming that the plasma is composed of protons only, and the total density obtained from CODIF, which is computed by summing the measured $\mathrm{H}^{+}$and $\mathrm{O}^{+}$densities, don't differ significantly. Indeed, while the composition of the beams is highly variable, oxygen ions rarely dominate the number density and the average composition of beams is $\sim 1 / 3$ of $\mathrm{O}^{+}$ and $\sim 2 / 3$ of $\mathrm{H}^{+}$. As a consequence, the presence of $\mathrm{O}^{+}$ions 

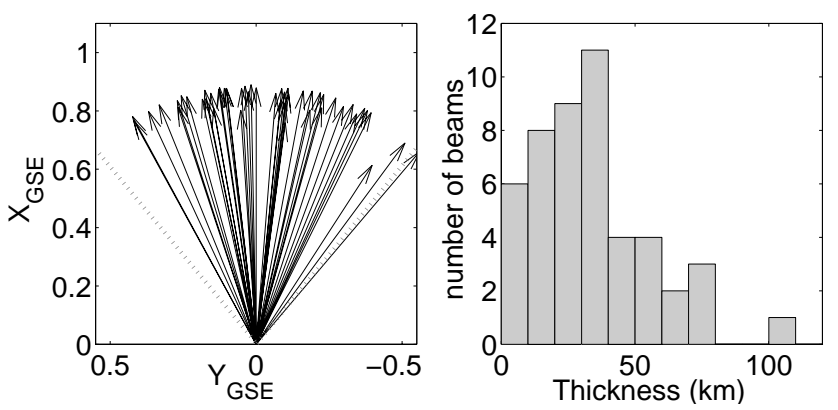

Fig. 4. Left panel: Projection in the $X Y_{\mathrm{GSE}}$ plane of the axis tangential to the ion beams and perpendicular to the magnetic field, evaluated from the MVA analysis of electric field data. The grey dotted lines delimit the region where the angle between this axis and the $X_{\mathrm{GSE}}$ axis is lower than $40^{\circ}$. Right panel: Ion beams thickness at ionospheric altitude $(100 \mathrm{~km})$.

in these beams may not alter significantly the density computed by HIA. Both CODIF and HIA reveal that the density rarely exceeds $0.2 \mathrm{~cm}^{-3}$. The average value given by these two experiments is respectively 0.097 and $0.091 \mathrm{~cm}^{-3}$. The plasma composition inside these beams is similar to the polar wind composition. Indeed, a statistical study by Su et al. (1998) using plasma composition measurements by the TIDE experiment onboard the POLAR spacecraft estimated the average proportion of $\mathrm{O}^{+}$at about $20 \%$ in the polar wind. Furthermore, the average cold ion density as estimated by Engwall et al. (2009) with Cluster in the lobe regions is $0.14 \mathrm{~cm}^{-3}$. PCIB differ from polar wind only by their temperature, which is typically of the order of tens of eV compared to $\sim 0.2-0.3 \mathrm{eV}$ for polar wind (e.g. Yau et al., 2007). It is actually not surprising as heating is expected both at low and high altitude. At ionospheric altitudes, plasma precipitation may excite lower hybrid waves in the upper ionosphere. These waves can lead to a transverse heating of the ionospheric ions by tens or hundreds of eV (Chang and Coppi, 1981). At higher altitudes, field aligned particle with different velocities are likely to excite instabilities that drive wave activity leading to ion heating (e.g. Bergmann et al., 1988). Maggiolo et al. (2006), and Reiff et al. (1988) have observed similar ion heating and noted the possibility that a two-stream instability may be involved. The PCIB thus look like heated and accelerated polar wind. This is confirmed by density estimations using data (not shown) from the WHISPER experiment for the event detected by Cluster on 18 May 2003 shown in Fig. 1. The plasma density can be estimated without bias with WHISPER by identifying characteristic plasma frequencies in wave spectrograms (Décréau et al., 2001). During this particular event, there is a good agreement between WHISPER and HIA/CODIF densities inside the acceleration regions. It indicates that the outflowing ionospheric plasma inside the beams is energetic enough to be accurately detected by the HIA and CODIF de- tectors. WHISPER density shows no significant differences of density inside and outside the beams while the HIA detects no ions in between the outflowing ion beams. Indeed, magnetospheric lobes are filled by polar wind plasma which is too cold to be detected by particle detectors because of the positive spacecraft potential. Thus, contrary to what is suggested by particle spectrograms, the outflow regions have similar plasma densities as the surrounding magnetospheric lobes, populated by cold polar wind plasma. The only difference is that in the outflow regions the plasma has been heated and accelerated enough so that it can be measured by particle detectors.

\subsection{Geometry of PCIB}

The orientation of the outflowing ion structures can be estimated by a minimum variance analysis applied to the electric field data. Polar cap ion outflow structures are likely to be elongated in the Sun-Earth direction and thin in the perpendicular direction, similar to polar cap arcs. An example has been shown by Teste et al. (2007) for the event presented in Fig. 1 of this paper.

We assume they can be described as planar structures parallel to the magnetic field lines surrounded by strong convergent electric fields perpendicular to the beam plane and to the magnetic field. We apply a minimum variance analysis (MVA) on electric field data. The axis of maximum variance corresponds to the direction of this strong convergent electric field. The associated PCIB direction is then given by the cross product between the maximum variance axis and the magnetic field direction.

For an accurate estimation of the MVA frame, the eigenvalues of the electric variance matrix must satisfactorily fulfill the condition $\lambda_{1} \gg \lambda_{2} \gg \lambda_{3}$ where $\lambda_{1}, \lambda_{2}$, and $\lambda_{3}$ are, respectively, the maximum, intermediate, and minimum variances. The three components of the electric field are computed by both EDI and EFW under the hypothesis that there is no electric field along the magnetic field. Thus, the minimum variance axis is always oriented along the magnetic field and $\lambda_{3}$ is always almost null and $\ll \lambda_{1}, \lambda_{2}$. Moreover, it implies that the intermediate variance axis gives the associated PCIB direction. In order to keep events where this direction is well estimated, we focus on structures where $\lambda_{1} / \lambda_{2}>10$. The results for the 50 beams satisfying these conditions are illustrated in the left panel of Fig. 4, which shows a projection of the intermediate variance axis in the $X Y_{\mathrm{GSE}}$ plane. All these vectors except one make an angle smaller than $40^{\circ}$ with the $X_{\mathrm{GSE}}$ direction. This implies that PCIB are mainly oriented in the Sun-Earth direction as are polar cap arcs (e.g. Valladares et al., 1994).

\subsection{Typical spatial scales of PCIB}

Figure $4 \mathrm{~b}$ shows the width of the beams at ionospheric altitude computed for the same subset of events. This width has 
been evaluated from the distance that the Cluster spacecraft traveled along the beam normal during the time it crosses it assuming that the PCIB didn't move significantly during that time. This assumption is supported by the relative stability of PCIB (see Sect. 3.7). If PCIB are the high altitude manifestation of polar cap arcs, the low drift velocity (less than $200 \mathrm{~m} \mathrm{~s}^{-1}$ at ionospheric altitude) reported by Valladares et al. (1994) also support the method used for width evaluation. The starting/ending times correspond to the moment when Cluster starts/finishes to detect upward accelerated ions. As the average spacecraft potential above the polar caps is $\sim 30 \mathrm{~V}$ and the low energy detection limit of the HIA experiment is $5 \mathrm{eV}$, the width we obtain is the size of the region where the outflowing ions have an energy higher than $\sim 35 \mathrm{eV}$. The thickness of all of the 50 PCIB of this subset of events is smaller than $100 \mathrm{~km}$. The distribution of the thickness peaks between 30 and $40 \mathrm{~km}$. This distribution is similar to the one obtained for the thickness of inverted-V events of precipitating auroral electrons measured by FAST (see Fig. 12 in Partamies et al., 2008) or by DMSP (peak at 28-35 km, Newell et al., 1996).

\subsection{Total ion outflow associated with PCIB}

The thermal speed of upgoing ions detected by Cluster is small compared to their bulk velocity. Consequently, there is little difference between single particle velocity, and plasma ion bulk velocity and the flux of outflowing particles from each species can be estimated by the product between the velocity and density. The $\mathrm{O}^{+}, \mathrm{H}^{+}$, and total ion flux mapped to ionospheric altitude (taken at $100 \mathrm{~km}$ altitude), are shown in Fig. 3d. Both species have been accelerated by a quasi-static electric field and gained the same parallel energy. Therefore, the heavier $\mathrm{O}^{+}$ions have parallel velocity 4 times lower than protons. Furthermore, the $\mathrm{H}^{+}$density is usually higher than the $\mathrm{O}^{+}$density. It results that $\mathrm{H}^{+}$clearly dominates the number flux of outflowing particles. The average flux of $\mathrm{O}^{+}\left(2.75 \times 10^{7} \mathrm{~cm}^{-2} \mathrm{~s}^{-1}\right)$ at ionospheric altitude is about 10 times smaller than the flux of $\mathrm{H}^{+}(4.45 \times$ $\left.10^{8} \mathrm{~cm}^{-2} \mathrm{~s}^{-1}\right)$. However, the mass flux associated with $\mathrm{O}^{+}$and $\mathrm{H}^{+}$outflows is approximately equal (respectively $7.31 \times 10^{-19} \mathrm{~kg} \mathrm{~cm}^{-2} \mathrm{~s}^{-1}$ and $7.39 \times 10^{-19} \mathrm{~kg} \mathrm{~cm}^{-2} \mathrm{~s}^{-1}$ at ionospheric altitude).

The total outflow associated with PCIB can be derived by multiplying the ionospheric flux by the cross-area at ionospheric altitude. As their average width at ionospheric altitudes is $\sim 37 \mathrm{~km}$ and the average total flux of particles is of about $4.85 \times 10^{8} \mathrm{~cm}^{-2} \mathrm{~s}^{-1}$, the average flux per unit of length is $\sim 1.8 \times 10^{17} \mathrm{~m}^{-1} \mathrm{~s}^{-1}$. Cluster data show that the beam length in the sunward direction can exceed $1000 \mathrm{~km}$ at Cluster altitude (e.g. Teste et al., 2007). As the mapping factor between Cluster and the ionosphere is about 10, the corresponding ionospheric length is about $100 \mathrm{~km}$ and the net ion outflow about $1.8 \times 10^{22} \mathrm{~s}^{-1}$. This corresponds to the average ion outflow associated with an isolated beam with av- erage characteristics and with a length corresponding to the maximum length observed by Cluster. However, the length observed by Cluster is limited by the spacecraft separation above the polar caps and the beams may be more elongated in the Sun-Earth direction. The average outflow associated with PCIB can be estimated accurately only if we can access the average length and the average number of beams, which is not possible with Cluster in-situ measurements. According to a statistical study by Ismail and Meng (1982), the typical length of isolated polar cap arcs in the Sun-Earth direction is about $1000 \mathrm{~km}$ at ionospheric altitude. If we suppose that PCIB are the high altitude signature of polar cap arcs and thus that both have similar size, we obtain $1.8 \times 10^{23} \mathrm{~s}^{-1}$ as the average outflow associated with an isolated PCIB. Multiple beams are frequently observed above the polar caps (up to 7 in our dataset). If we suppose that there are 5 beams in each hemisphere, we estimate that the average outflow due to PCIB is of the order of $\sim 10^{24} \mathrm{~s}^{-1}$. Note that this estimation doesn't take into account beams with low parallel velocities that are not included in our statistic. According to the PCIB energy distribution (Fig. 3a) they are likely to be more frequent than high energy beams. However, the particle flux in these beams should be low and thus taking them into account shouldn't significantly change the outflow estimation.

We can also estimate the upper boundary for the total outflow associated with polar cap arcs. As shown in Fig. 3d, the maximum particle flux at ionospheric altitude is $\sim 10^{9} \mathrm{~cm}^{-2} \mathrm{~s}^{-1}$. This corresponds to beams with relatively high energies and densities. The maximum width of a beam is of the order of $100 \mathrm{~km}$ at ionospheric altitude. The maximum outflow per unit of length is then of about $\sim 10^{18} \mathrm{~m}^{-1} \mathrm{~s}^{-1}$. The maximum length for a beam would be reached if it crosses the entire polar cap. If we assume the polar cap boundary is located at $75^{\circ}$ magnetic latitude, the corresponding length would be about $5500 \mathrm{~km}$ at ionospheric altitude. In that case, the outflow would be $5.5 \times 10^{24} \mathrm{~s}^{-1}$. Thus, in the case when 10 dense and energetic PCIB cross the whole polar cap, the maximum outflow above the northern polar cap would be of about $5.5 \times 10^{25} \mathrm{~s}^{-1}$ and the total outflow including both hemispheres would be of about $10^{26} \mathrm{~s}^{-1}$. We estimate that this value represents the upper boundary of the outflow associated with PCIB. It is of the order of magnitude of high latitude cold ion outflow $\left(10^{26} \mathrm{~s}^{-1}\right)$, as recently estimated by Engwall et al. (2009) with the EFW experiment onboard Cluster for ions in the $0-60 \mathrm{eV}$ range. However, the estimated average outflow due to PCIB $\left(\sim 10^{24} \mathrm{~s}^{-1}\right)$ is 2 orders of magnitude lower. This ionospheric ion source is much more localized than the polar wind, which covers the whole polar cap. Furthermore, this is an intermittent source of plasma, only active after prolonged periods of northward IMF. The consequence is that locally accelerated PCIB are a minor source of ionospheric plasma compared to other continuous sources like the polar wind except during specific periods. However, it may be more significant in terms of net ionospheric plasma loss. According to Seki et al. (2001), the 

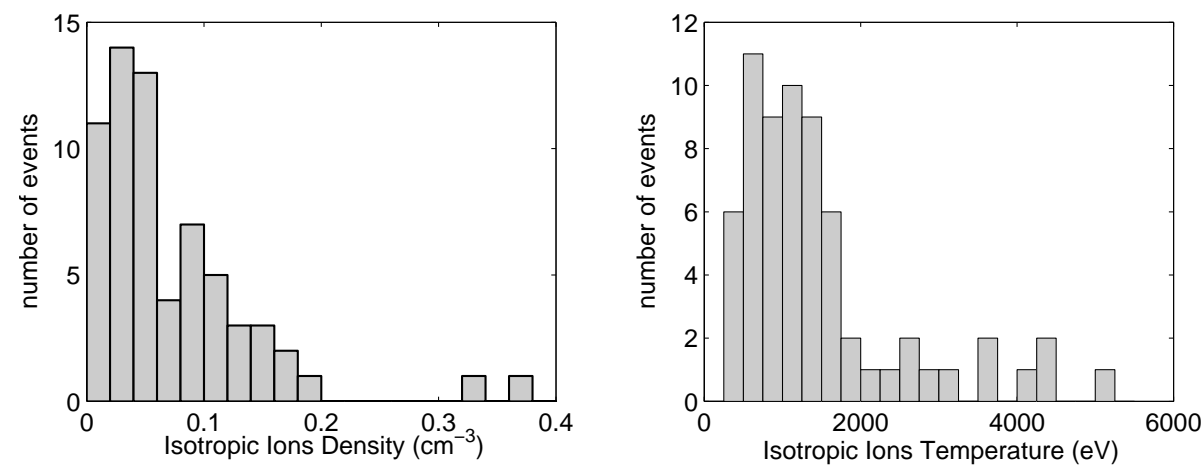

Fig. 5. Properties of the isotropic population coincident with Type I polar cap ion beams as computed from HIA. Left panel: isotropic ion density. Right panel: isotropic ion temperature.

total oxygen loss rate is on average about one order of magnitude lower than the polar $\mathrm{O}^{+}$outflow rate. This difference is due to a substantial return flux from the magnetosphere to the ionosphere. Seki et al. (2001) estimated the net oxygen ion loss to $\sim 5.10^{24} \mathrm{~s}^{-1}$. During periods of northward IMF, PCIB can thus possibly contribute the same order of magnitude to oxygen loss. It depends on whether PCIB escape into the interplanetary medium or are finally returned to the ionosphere. Thus, determining PCIB contribution to the net loss of ionospheric ions requires a good knowledge of ion trajectories in the magnetotail. This may be the subject of another study including a forward tracing of PCIB that takes into account the magnetic field and convection topology during prolonged periods of northward IMF.

\subsection{Background isotropic population associated with PCIB}

An interesting feature of these ion beams is that the ouflowing ions are frequently accompanied with a background population of magnetospheric ions. We identified two types of PCIB: Type I are upflowing ion beams collocated with an isotropic ion population, and Type II beams for which the isotropic component is absent. Type I beams were found in $40 \%$ of the analyzed events. The temperature and density distributions of the isotropic component in Type I PCIB are displayed in Fig. 5. Their temperature ranges from $300 \mathrm{eV}$ to $\sim 3 \mathrm{keV}$ while their density is quite low and highly variable. This background population is isotropic and no significant fluxes of $\mathrm{O}^{+}$ions are detected by the CODIF experiment, indicating it consists almost exclusively of protons. However, as the isotropic ion density can be very low during some events, it is possible that there is a nonzero $\mathrm{O}^{+}$contribution but that the flux is simply too low to produce significant count rates in the CODIF detector.

PCIB could in principle be the source of this isotropic population if part of this population is heated and pitch-angle scattered by waves. However, this seems unlikely. Indeed, contrary to $\mathrm{PCIB}$, no $\mathrm{O}^{+}$ions are detected in the isotropic population. Any scattering process that could transform PCIB into the isotropic population must only act on $\mathrm{H}^{+}$ions. There is no evidence of such wave activity.

In addition, the background population has higher temperatures than typical mantle and magnetosheath temperatures, and contrary to them is isotropic with no significant bulk velocity. Thus, the more likely source for this background population is magnetospheric plasma, i.e. a plasma population that stayed enough time in the magnetosphere to be isotropized and heated to the observed temperatures. This isotropic population has pitch angle distributions and temperatures similar to the plasmasheet. However, it is slightly colder than typical plasmasheet plasma which is not surprising as during prolonged periods of northward IMF the plasma sheet gets colder and denser because of increased penetration of solar wind plasma into the magnetospheric tail (e.g. Fujimoto et al., 1996; Terasawa et al., 1997; Wing and Newell, 1998). The temperature of the Cold Dense Plasma Sheet (CDPS), $1 \mathrm{keV}$ or less (e.g. Fujimoto et al., 1996), corresponds to the temperature of the background protons we measure. The CDPS is depleted in $\mathrm{O}^{+}$because of its solar wind origin and thus the lack of $\mathrm{O}^{+}$in the isotropic background population is consistent with this hypothesis. However, the background proton density is lower than the typical CDPS density. It ranges from almost 0 to $0.2 \mathrm{~cm}^{-3}$ while CDPS density can exceed $1 \mathrm{~cm}^{-3}$ (e.g. Fujimoto et al., 1996; Thomsen et al., 2003). Note that we have found no correlation between the temperature and density of the background isotropic ion population and that its kinetic pressure is always low compared to the magnetic pressure. Cluster data reveals a rather broad range of situations for the isotropic population: dense and hot, dense and cold, tenuous and dense or tenuous and cold, while the velocity distribution function of these ions is always isotropic. This low and variable density may be linked to the process that brings these ions at such high latitude above the polar caps or to a temporal evolution or dissipation of this population once it has been brought there. It can also depend on the region of the plasma sheet where these ions originate, as plasma sheet density 

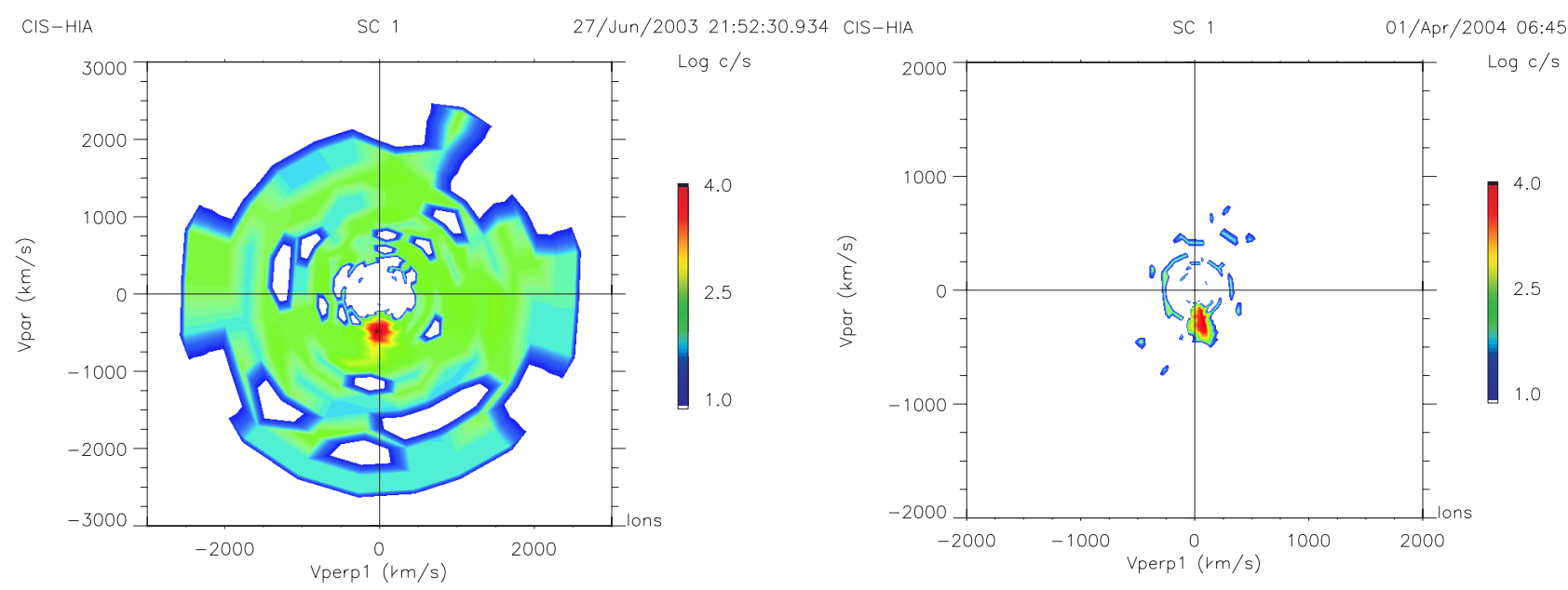

Fig. 6. Bi-dimensional cut of the ion distribution function in the $V_{\text {perp }}-V_{\text {par }}$ plane for spacecraft 1 on 27 June 2003 around 21:52:30 UT (left panel) and on 1 April 2004 around 06:45 UT (right panel). $V_{\text {perp }}$ and $V_{\text {par }}$ are defined with respect to the local magnetic field.

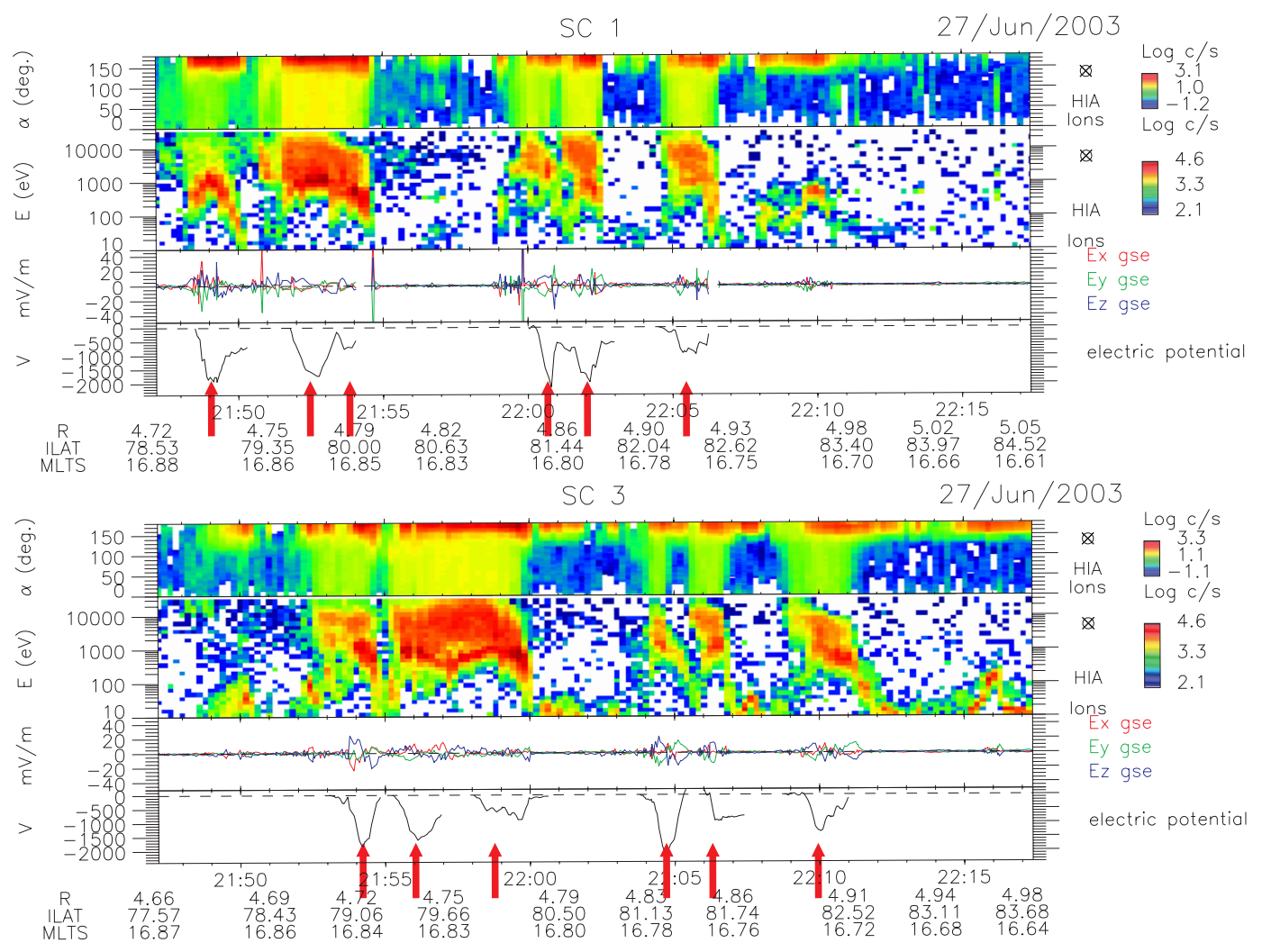

Fig. 7. 27 June 2003, data from Cluster 1 and 3. For each spacecraft, from top to bottom: ion pitch angle distribution from HIA for all ions; time-energy spectrogram from HIA for all ions; GSE components of the perpendicular electric field as given by EFW; electric potential along the spacecraft trajectory computed from EFW data. The red arrows show the location of the ion beams detected by each spacecraft. Note also the clear electric signatures of the PCIB.

varies with tailward distance and distance to the flanks (e.g. Wing and Newell, 1998). However, no significant correlation has been found between the MLT and Invariant Latitude where the background population was detected and its tem- perature and density. As they occur at high latitude, PCIB may preferentially lie on magnetic field lines mapping in the far tail where ion density and $\mathrm{O}^{+}$proportion should be low and thus where the plasma sheet characteristics (in particular 
density and composition) may be more similar to that of the background isotropic ions. As PCIB are observed during prolonged periods of northward IMF (see Sect. 4.2), we suggest that the CDPS may be the source of the background population.

Figure 6 shows an example of two bi-dimensional cuts of the ion velocity distribution function in the $V_{\perp}-V_{/ /}$ plane. The first example is taken on 27 June 2003 around 21:52:30 UT (2nd beam in Fig. 7) and illustrates a Type I ion beam. It reveals the presence of two ion populations: a cold beam with a small perpendicular velocity, accelerated in the direction antiparallel to the magnetic field; and relatively hot and dense background isotropic ions. The second event in Fig. 6 illustrates a Type I polar cap beam detected on 1 April 2004 around 06:45 UT (see Fig. 2). Two populations, the beam and the isotropic ions, are again observed. During this event, the background population is cold and particularly tenuous but it is still isotropic. Figure 6 illustrates that independently of their temperature or density the velocity distribution function of the background population is always very close to isotropy.

Note also that the hot isotropic protons are detected precisely in the same regions as the outflowing ion beams (see Figs. 1, 2, and 7). For the Type I ion beams, the outflow region is limited to the region where the background population is detected. It thus seems that isotropic ions play a role in the processes that generate the Type I PCIB and that the size of the Type I PCIB is related to the size of the isotropic background plasma clouds. However, the majority of the beams $(60 \%)$ are of type I - with no isotropic background - and type I and II ion beams have similar energy, size, density, and composition distributions.

Several configurations are thus possible and the distinction between Type I and Type II PCIB is arbitrary, as it depends on the sensitivity of the ion detector. This complicated situation is well illustrated by the polar cap crossing displayed in Fig. 1. Cluster is crossing the Northern polar cap from $\sim 83^{\circ}$ latitude to $\sim 88^{\circ}$ latitude in the 18:00-19:00 MLT sector. The first Type I beam is detected around 11:12 UT; it shows a tenuous background population of isotropic protons with a temperature of $\sim 2 \mathrm{keV}$ and a very low density (less than $0.002 \mathrm{~cm}^{-3}$ ). Around 11:16 UT Cluster detects a smaller Type II beam. Then, from 11:27 UT to 11:35 UT, data show a third outflowing ion beam, of Type I, accompanied by an intense isotropic population. Note that around 11:30 UT one can identify a sharp transition in the energy spectra of the isotropic population accompanying the beam: the isotropic ion population is colder before 11:30 UT $(500 \mathrm{eV})$ and hotter after $(\sim 1000 \mathrm{eV})$. The isotropic plasma cloud is detected together with a broad ion beam, seemingly composed of two merged inverted V structures. Then, around 11:40 UT a fourth beam is detected. This Type II beam also comprises two substructures but is not accompanied by a background population. The more energetic ion beam $(700 \mathrm{eV})$ is the one associated with the more intense background popula- tion. However, the last beam, not associated with isotropic protons, is more energetic than the first one, which is measured together with a tenuous population of isotropic protons (respectively $500 \mathrm{eV}$ and $150 \mathrm{eV}$ ).

The study of the processes that bring isotropic plasma at high latitudes above the polar cap is of great interest to understanding the dynamics of the magnetospheric lobes and of the magnetotail during prolonged periods of northward IMF. This is a key question to address in order to understand how polar cap current systems are generated. However, it is beyond the scope of this paper and it will only be briefly discussed in the last section.

\subsection{Lifetime of PCIB}

As stated by Maggiolo et al. (2006) and Teste et al. (2007), the PCIB measured during the 18 May 2003 event displayed in Fig. 1 are stable and their lifetime is longer than $30 \mathrm{~min}$. A comparison between different Cluster data for the events of our dataset reveals that the beams are indeed usually stable with long lifetimes. Similar structures are frequently observed on the different Cluster spacecraft with a delay of several minutes. The maximum delays (up to $30 \mathrm{~min}$ ) are observed when the Cluster spacecraft separation distance is large (i.e. during the first half of 2003 and in 2006). This is exemplified in Fig. 7, which shows data from Cluster 1 and 3 for a polar cap crossing on 27 June 2003. During this polar cap crossing, 6 ion beams and associated electric potential structures are observed by both spacecraft. Despite some small variations, there is no doubt that these 2 spacecraft cross the same beams with a delay of about $5 \mathrm{~min}$. These beams are approximately detected at the same location and no significant drift of the structures occurred during this 5 min period. The long lifetime of the structures confirms the acceleration by quasi-static electric fields. Furthermore, it is consistent with the polar cap arc lifetime, which can exceed one hour and is typically tens of minutes (see Zhu et al., 1997). As it can be seen in Fig. 7, not only the beams are long-lived but also the isotropic ion clouds that can accompany them. When comparing data from different Cluster spacecraft we were not able to identify any significant time evolution of the isotropic plasma clouds in our dataset. Their density and temperature remain virtually the same between successive Cluster observations; the general trend is that the properties of the isotropic plasma remain almost unchanged on a time scale of several minutes.

\section{PCIB dependence on solar wind and IMF}

This section discusses the dependence of PCIB on interplanetary parameters like solar wind pressure and density and interplanetary magnetic field (IMF) orientation. Solar wind parameters from the ACE spacecraft were shifted to 1 AU. IMF components are given in the GSM frame. The results are 
compared to the dependence of polar cap arcs on interplanetary parameters as given by previous studies using optical data both from ground and spacecraft imagers. Most of these studies are discussed in a review paper on polar cap arcs by Zhu et al. (1997).

The Cluster observations analyzed in this study do not show a clear correlation between the occurrence of polar cap outgoing ion beams and the solar wind density, velocity, dynamic pressure, or IMF magnitude.

\subsection{PCIB dependence on IMF $B_{X}$ and $B_{Y}$}

Figure 8 shows how the PCIB occurrence rate varies with the IMF components in the GSM frame. The grey bars include all events while the red (green) bars correspond to beams associated (or not associated) with hot isotropic ions.

The beams are preferentially detected during periods of negative $B_{\mathrm{X}}$ (median value $-1.5 \mathrm{nT}$ ), However, this is actually true only for Type II beams (median value $-2.3 \mathrm{nT}$ ); indeed the occurrence of Type I PCIB doesn't seem to depend on IMF $B_{\mathrm{X}}$ (median value $0.58 \mathrm{nT}$ ). Lassen and Danielsen (1978) and Kullen et al. (2002) reported that polar cap arc occurrence is influenced by the IMF $B_{\mathrm{X}}$, suggesting that more polar cap arcs are observed during periods of negative $B_{\mathrm{X}}$. Other studies, however, found no clear correlation between the occurrence of polar cap arcs and the IMF $B_{X}$ (e.g. Ismail et al., 1977; Gussenhoven, 1982).

PCIB are mainly observed during periods of positive IMF $B_{\mathrm{Y}}$ (median value $1.6 \mathrm{nT}$ ). This is true for both Type I (median value $1.57 \mathrm{nT}$ ) and Type II (median value $1.7 \mathrm{nT}$ ) ion beams. Several studies (e.g. Gussenhoven, 1982; Elphinstone et al., 1990; Makita et al., 1991) reported that polar cap arcs are preferentially detected in the evening sector for $B_{Y}$ positive. The MLT distribution of PCIB detected by Cluster (Fig. 9) is consistent with this result. While in the morning sector the beams are approximately detected as often for positive or negative $B_{Y}$, in the evening sector most of the beams are detected for positive $B_{Y}$. Furthermore, it appears that in our dataset, there are much more beams located in the evening side than in the morning side. The cause of this asymmetry is not clear.

As stated in the review by Zhu et al. (1997), while IMF $B_{\mathrm{X}}$ and $B_{\mathrm{Y}}$ seem to influence the location and motion of polar cap arcs, there still exists some controversy on their effects. Analyzing the influence of IMF on the location of PCIB is not straightforward, as the orbit of Cluster spacecraft influences the location of beams detection (see Sect. 5). In addition, it must be noted that due to the orientation of the Parker spiral, IMF $B_{\mathrm{X}}$ and $B_{\mathrm{Y}}$ are dependant. Consequently, positive IMF $B_{\mathrm{Y}}$ is statistically more often associated with negative IMF $B_{\mathrm{X}}$. These biases prevent us from obtaining unambiguous conclusions about the IMF $B_{\mathrm{X}}$ and $B_{\mathrm{Y}}$ control on PCIB. We can only state that during periods of positive IMF $B_{Y}$ we detect more beams in the evening sector.
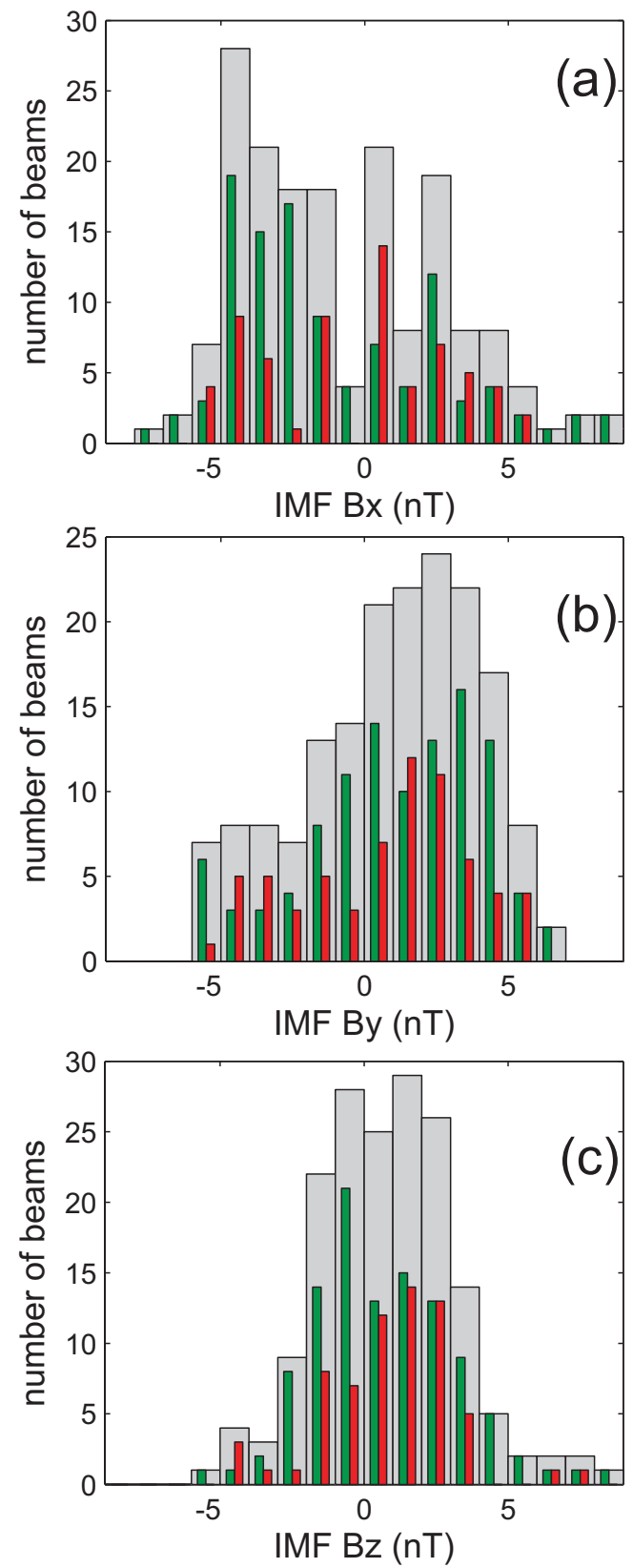

Fig. 8. Summary of the role of IMF on the occurrence of PCIB. IMF components are given in the GSM frame. From top to bottom: occurrence frequency of PCIB as a function of IMF $B_{X}, B_{Y}$ and $B_{\mathrm{Z}}$. For each panel, the red bars show the distribution for Type I ion beams, the green for Type II ion beams, and the grey bars for all the ion beams. The IMF $B_{Z}$ is obtained from the ACE spacecraft and is shifted to $1 \mathrm{AU}$.

\subsection{PCIB dependence on IMF $B_{Z}$}

There is less controversy on the relation between IMF $B_{\mathrm{Z}}$ and polar cap arcs. Polar cap arcs appear during quiet geomagnetic activity periods (e.g. Davis, 1963; Ismail et al., 1977). They are thus preferentially observed during periods 


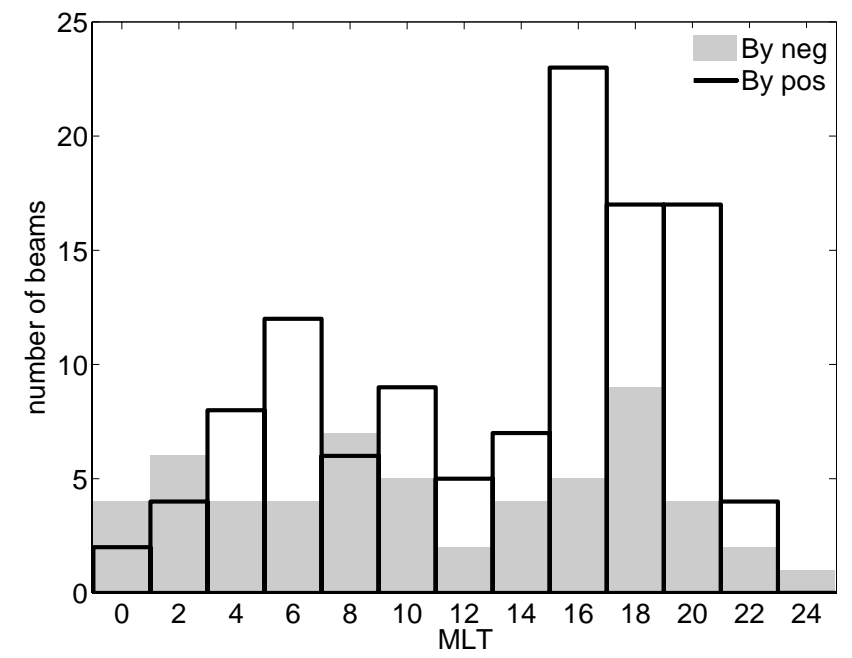

Fig. 9. The distribution of polar cap ion beams as a function of magnetic local time (MLT). The white bars correspond to events detected during periods of positive IMF $B_{Y}$ and the grey ones for events detected during periods of negative $B_{\mathrm{Y}}$. The mapping uses the Tsyganenko 89 model (Tsyganenko, 1989).

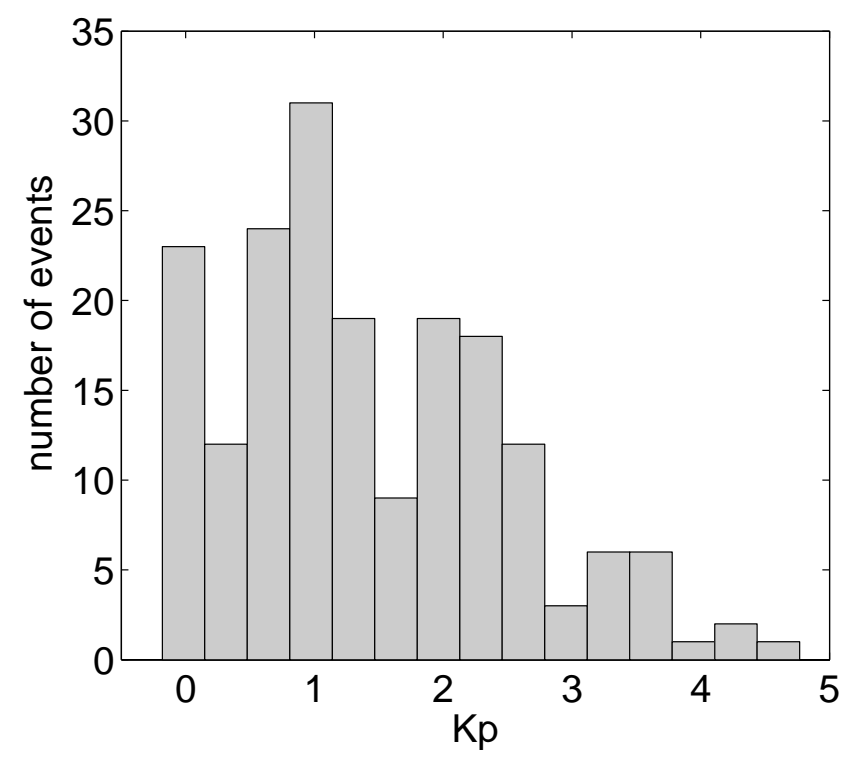

Fig. 10. The occurrence frequency of PCIB as a function of the Kp index.

of northward IMF. However, they can also be observed during southward IMF as reported by Rairden and Mende (1989) and Valladares et al. (1994), who respectively found that 67$80 \%$ of polar cap arcs occur for $\mathrm{B}_{Z}$ north conditions. We observe a similar behavior for PCIB. They are preferentially detected during periods of low geomagnetic activity with low Kp (Fig. 10) and the majority of them (65\%) - but not all are detected during $B_{\mathrm{Z}}$ north conditions.

Figure 11 shows the evolution of the percentage of PCIB events detected when $B_{\mathrm{Z}}$ is positive as a function of the time

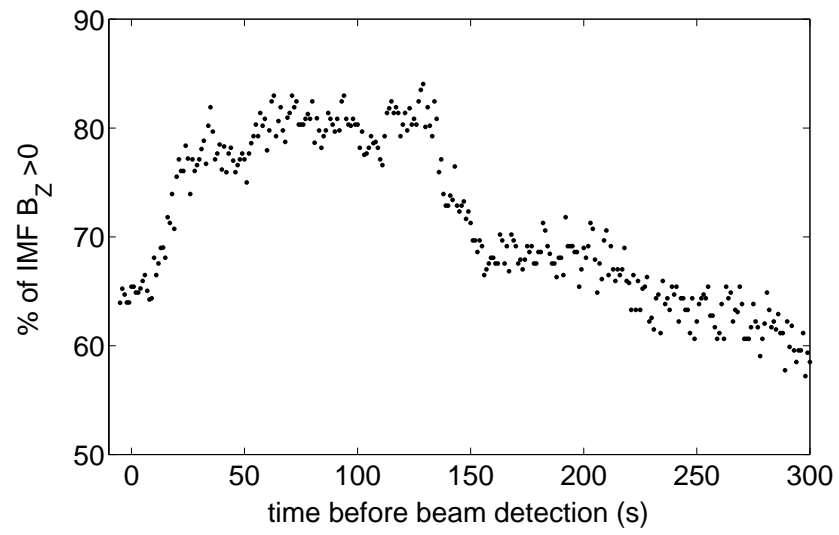

Fig. 11. The figure illustrates the percentage of events for which the IMF $B_{Z}$ component in the GSM frame was positive as a function of a time lag, $\Delta t$, prior to the PCIB detection by Cluster. The IMF $B_{Z}$ is obtained from the ACE spacecraft and is shifted to $1 \mathrm{AU}$.

lag between the beam detection and the moment when the $B_{\mathrm{Z}}$ sign is measured. This plot was obtained by checking the orientation of IMF $B_{Z}$ for a variable time lag $\Delta t$ prior to the event for all the events in the database. Figure 11 shows the results for $\Delta t$ taking values between 0 and $300 \mathrm{~min}$; it evidences that from $\sim 20 \mathrm{~min}$ to $\sim 2 \mathrm{~h}$ before the beam detection, the probability of having positive $B_{\mathrm{Z}}$ is the highest $(\sim 80 \%)$. PCIB are thus detected after prolonged periods of northward IMF. However, a large fraction of them (35\%) is detected while IMF $B_{\mathrm{Z}}$ is oriented southward. It implies that there should be a delay between a northward/southward turning of the IMF and their appearance/disappearance.

From two hours to five hours before detection of PCIB, the probability of having $B_{\mathrm{Z}}$ positive decreases to $50 \%$. Thus, for the majority of the events of our dataset, the IMF turned northward more than $2 \mathrm{~h}$ prior to the detection of the beams. We conclude that the delay between a northward turning of the IMF and the appearance of beams is about $2 \mathrm{~h}$. Then, between $20 \mathrm{~min}$ and $2 \mathrm{~h}$ prior to the beam detection, there is a plateau with a high probability of having $B_{\mathrm{Z}}$ north. This probability sharply decreases for $\Delta t$ between 0 and $20 \mathrm{~min}$ prior detection of beams. Thus, for a significant part of the events of our dataset, $B_{\mathrm{Z}}$ turned southward less then $20 \mathrm{~min}$ before but the beams are still observed above the polar caps. Consequently, the delay between a southward turning of the IMF and the disappearance of beams may be of the order of $20 \mathrm{~min}$. Note that we obtain comparable $B_{\mathrm{Z}}$ profiles and time delays when we repeat this analysis for PCIB of Type I and II taken separately. These delays are similar to the delay between polar cap arc appearance/disappearance and $B_{\mathrm{Z}}$ turnings. In the study of Kullen et al. (2002), the highest probability of having northward IMF was found 1 to $2 \mathrm{~h}$ before polar cap arc detection. The delay between a northward turning of the IMF and the appearance of polar cap arcs has been estimated to be $1 \mathrm{~h}$ (Troshichev et al., 1988). On the other 
hand, the estimated delay between a southward turning of the IMF and the disappearance of polar cap arcs varies from 10-15 min (Troshichev et al., 1988) to 30 min (Valladares et al., 1994; Rodriguez et al., 1997).

As suggested by Valladares et al. (1994) for polar cap arcs, the delays between IMF turnings and the appearance/disappearance of PCIB may be related to the time needed for the magnetosphere to complete or lose its northward IMF configuration. Thus, PCIB and the processes that lead to their formation appear to be a feature of the global magnetospheric configuration during prolonged periods of northward IMF.

\section{Location of PCIB}

\subsection{Hemispheric asymmetry of the observations}

Most of the events of our dataset occur at altitudes comprised between 3 and $6 R_{\mathrm{E}}$. The highest altitude where a beam has been identified is $7.8 R_{\mathrm{E}}$. There are several reasons that may explain why we don't detect beams at higher Cluster altitudes. The data selection criteria (A.2 and $\mathrm{B}^{\prime}$, see Sect. 2) identify the PCIB based on the local value of the density; therefore these criteria may favor detection of PCIB at low altitudes where the density inside the outgoing ion beams is higher. Due to the divergence of magnetic field lines, the more the altitude increases, the lower the density is inside the beams. Thus, at high altitudes, the density inside the ion beams is less likely to satisfy the criteria defined for the automated search $\left(N>0.03 \mathrm{~cm}^{-3}\right)$ in the AMDA database. Moreover, at high altitude the intensity of the converging electric field structures associated with ion beams becomes less intense because of the magnetic field line divergence. Thus, the electric signature of PCIB is more difficult to identify, which will again favor the detection of beams at lower altitudes. At higher altitudes, above the acceleration region, the ion beams may also be dispersed by the cross-field convection and become destructured by other processes like centrifugal acceleration or wave particle interactions. The altitude dependence of beam detection has consequences on the hemispheric distribution of events in our dataset. While above the Northern polar cap Cluster samples altitudes down to $\sim 3 R_{\mathrm{E}}$, it almost never samples altitudes below $5 R_{\mathrm{E}}$ above the Southern polar cap. Because the beams are preferentially detected at low altitude, it results that the majority of the events of our dataset (92\%) are located in the Northern Hemisphere.

\subsection{Ionospheric mapping of PCIB}

Figure 12a shows the location of the footpoints of magnetic field lines on which PCIB have been detected (in red for Type I beams and in green for Type II). The mapping has been done using the Tsyganenko 89 magnetic field model (Tsyganenko, 1989) and the ionospheric level is taken to be at $100 \mathrm{~km}$ altitude. PCIB can be detected everywhere above the polar caps. While the auroral zone extends to higher latitude for low Kp, the latitude where PCIB occur is not correlated to Kp. Furthermore, PCIB can be detected at very high latitudes, indicating that they are not necessarily located adjacent to the auroral oval but can actually be located everywhere above the polar caps. This is confirmed by Cluster particle spectrograms which clearly show that PCIB can be detected everywhere in the lobe region, far from the plasmasheet.

The distribution of PCIB above the polar caps is not uniform. As seen in Fig. 9, more beams are detected in the dusk sector than in the dawn sector. Furthermore, only a few beams are detected around noon and midnight. However, the Cluster spacecraft don't provide a uniform coverage of the polar caps as their altitude varies when they cross them. The perigee of the Cluster spacecraft moves around the Earth in the course of the year. Consequently, it is located in the nightside during winter, in the dayside during summer, in the dawnside during spring and in the duskside during autumn. Thereby, during spring, the Cluster spacecraft will cross the northern polar cap from dawn to dusk and its altitude will increase while it moves across it. As discussed in Sect. 5.1, the probability to detect PCIB depends on the Cluster altitude, thus during spring, the probability of detecting a polar cap ion beam will be higher on the dawn side than on the dusk side. Furthermore, Cluster perigee is not in the ecliptic plane. It is actually slightly shifted in the Northern Hemisphere, complicating the situation even more. The geometry of the beams could also introduce a bias. The PCIB, as the polar cap arcs, tend to be elongated in the Sun-Earth direction and they are quite thin in the dawn-dusk direction. As a consequence, the probability of crossing a beam is higher during spring and autumn when the Cluster spacecraft move roughly in the dawn-dusk direction, i.e. perpendicular to the PCIB and polar cap arcs. This may partly explain why more beams are detected in the dawn and dusk sectors than in the noon and midnight sectors. The latitudinal distribution of beams peaks around 85 degrees, which corresponds to the latitude that is best sampled by the Cluster spacecraft in the altitude range where we detect PCIB. It indicates that the Cluster orbit influences the spatial distribution of the events of our dataset. Because of the bias caused by the orbit of Cluster we cannot give a definitive conclusion about the spatial distribution of PCIB. However, the comparison between the location of the ion beams with and without an associated background isotropic population reveals that both cases can be encountered everywhere above the polar cap; however, Type II PCIB, not associated with isotropic ions, tend to be located at higher latitudes than Type I PCIB. 

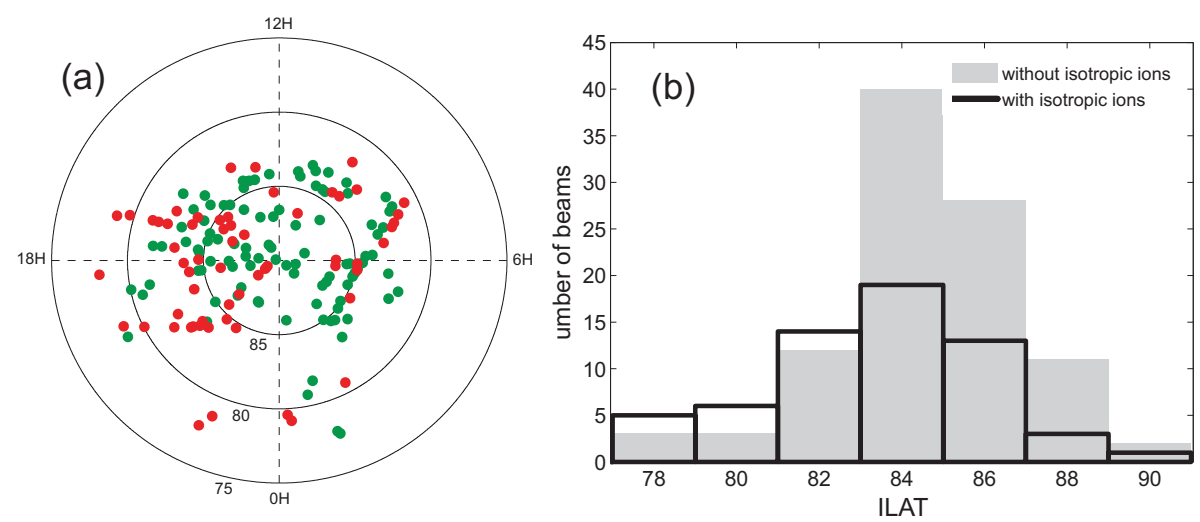

Fig. 12. (a) Ionospheric footpoints of the magnetic field lines on which polar cap ion beams are detected. Red dots stand for Type I ions beams; green dots correspond to Type II ion beams. (b) The distribution of polar cap ion beams as a function of invariant latitude (ILAT). The white bars correspond to Type I PCIB and the grey bars to Type II PCIB. The mapping was made using the Tsyganenko 89 model (Tsyganenko, 1989).

\section{Summary and discussion}

In this paper we have discussed Cluster observations of ion beams with an inverted- $\mathrm{V}$ shape detected above the polar caps, at altitudes comprised between $\sim 3$ and $7.8 R_{\mathrm{E}}$. The events were identified with the AMDA tool using an automated procedure. The Cluster observations show that ions originating from the polar ionosphere are accelerated along the magnetic field lines by quasi-static field-aligned electric fields. Our statistical study uses a dataset of 185 events of which $92 \%$ are located above the Northern polar cap. It provides a detailed description of their properties that can be summarized as follows:

1. PCIB are associated with quasi-static converging electric fields. The average field-aligned accelerating potential drop is estimated to be about $370 \mathrm{~V}$; a maximum value of about $2 \mathrm{kV}$ has been observed.

2. The average density of upflowing ions is about 0.09 $0.1 \mathrm{~cm}^{-3}$. The ion beams are composed of $\mathrm{H}^{+}$and $\mathrm{O}^{+}$ with varying proportions. While sometimes the $\mathrm{O}^{+}$can dominate, the average proportion is $2 / 3$ of $\mathrm{H}^{+}$and $1 / 3$ of $\mathrm{O}^{+}$. These ions look like heated and accelerated polar wind.

3. The ion beams are structures elongated in the Sun-Earth direction. At ionospheric altitudes, their width is usually less than $100 \mathrm{~km}$ with a typical spatial scale of about $30-40 \mathrm{~km}$.

4. The PCIB are not a major source of ionospheric plasma escape. It is an intermittent source active only during prolonged periods of northward IMF. The average particle number flux at ionospheric level is about $4.85 \times 10^{8} \mathrm{~cm}^{-2} \mathrm{~s}^{-1}$ and the average total ion outflow about $10^{24} \mathrm{~s}^{-1}$. However, the maximum outflow asso- ciated with PCIB can approach $10^{26} \mathrm{~s}^{-1}$, the estimated outflow rate of the main ionospheric sources.

5. The PCIB occur during periods of low geomagnetic activity (typically $\mathrm{Kp}<3$ ). While only $65 \%$ of them were observed during northward IMF, most of them $(80 \%)$ occur after prolonged periods of northward IMF. The delay between a northward turning of the IMF and their appearance is estimated to be approximately $2 \mathrm{~h}$; conversely a southward turning of the IMF seems to trigger the disappearance of the beams after roughly $20 \mathrm{~min}$.

6. The PCIB are preferentially observed during periods of positive IMF $B_{Y}$. During periods of positive IMF $B_{Y}$, PCIB are preferentially detected in the evening sector.

7. The analysis identified two types of upgoing ion beams: Type I represents $40 \%$ of the PCIB and corresponds to beams embedded into a hot isotropic ion population. Detection of this background population correlates well with detection of the beams; its density is highly variable, from almost 0 to $0.2 \mathrm{~cm}^{-3}$, and its temperature ranges from a few hundred $\mathrm{eV}$ to $2 \mathrm{keV}$. Type II ion beams are not accompanied by an isotropic ion component. Type I and II PCIB have similar statistical properties except that Type II tend to be located at higher latitude than Type I PCIB.

8. The PCIB are long-lived and stationary structures with lifetimes of several minutes at least.

9. There is a tendency of PCIB to be located towards the flanks of the polar caps; however, this effect needs further investigation as it may depend on the characteristics of the Cluster orbit and the event selection criteria.

PCIB are associated with field-aligned potential drops about one order of magnitude lower that the typical field-aligned 
potential drops associated with auroral zone inverted-Vs, but have similar widths.

The statistical properties of PCIB show similarities with polar cap arcs observed with ground and spacecraft imagers. The PCIB and polar cap arcs both show the same dependence on IMF $B_{\mathrm{Z}}$, the same geometry, i.e. thin in the dawndusk direction and elongated in the Sun-Earth direction, and have similar lifetimes. PCIB are accelerated upward by a field-aligned electric field that also accelerates electrons in the downward direction. Precipitating electrons in PCIB collide at lower altitudes with ionospheric particles generating photo-emissions like in polar cap arcs. Furthermore, according to Cluster data, PCIB are clearly separated from the plasma sheet and therefore they are detached from the auroral oval. The lower latitude parts of these structures may be connected to the auroral oval; however they are clearly a polar cap phenomenon. It is thus likely that PCIB and polar cap arcs are different aspects of the same magnetospheric processes. We interpret the PCIB Cluster events detected by Cluster as the high-altitude signature of the lower altitude polar cap arcs. Additional evidence should be provided by correlated campaigns including spacecraft and ground-based observations.

Cluster data at relatively high altitudes can shed new light on the study of polar cap arcs. At higher altitudes, due to the divergence of magnetic field lines, the structures get wider and thus allow Cluster to obtain more detailed measurements. The field-aligned potential drop associated with PCIB is quite low. The precipitating electrons have relatively low energies and may not always be able to trigger a significant luminous emission. It is therefore possible that, depending on the detector sensitivity, optical observations reflect only a fraction (the more energetic events) of polar cap arcs. This should be taken into account when interpreting polar cap arc optical observations. Furthermore, the Cluster spacecraft observe the magnetospheric lobes closer to the generator/driver of polar cap arcs and can bring new data on the latter.

The strong spatial correlation between the PCIB and the presence of an isotropic hot ion population suggests that the two are related to the same mechanism driving field-aligned acceleration above the polar cap during/after prolonged periods of northward IMF. The temperature and the pitch angle distribution of the isotropic component suggest that its origin may be the Cold Dense Plasma Sheet. A more detailed analysis is necessary to clearly identify the source of this isotropic plasma and the processes that bring it into the magnetospheric lobes. This is beyond the scope of this paper.

The reason why only a fraction of PCIB is accompanied by the isotropic component, even during the same polar cap crossing, is not clear. The two classes of beams, Type I and II, can correspond to different classes of beams generated by distinct processes. It is also possible that isotropic ions are always associated with PCIB but that sometimes they are not detected by the Cluster spacecraft, for example because of a temporal evolution of this population or because it is too tenuous at Cluster altitude to be measured. The fact that all beams have similar statistical properties and that the isotropic ion density is highly variable and reaches extremely low values (at the limit of the detection threshold of Cluster particle detectors) seems to favor the second hypothesis.

The Cluster multi-point measurements clearly show that the isotropic plasma filaments we observe are actually spatial structures. Indeed, the detection of isotropic plasma filaments associated with PCIB at similar location and with a delay of several minutes by different Cluster spacecraft exclude that they are temporal features associated with the flapping motion of the plasma sheet.

The presence of plasma material inside the lobes has been reported previously, for example from Interball data. Koleva and Sauvaud (2008) discussed observations of mixed plasma sheet/magnetosheath material and of plasma sheet structures characteristic of the cold plasma sheet inside the lobes. Grigorenko et al. (2002) reported Interball observations of plasma sheet filaments inside the lobes during northward IMF conditions and closer to the PSBL during southward IMF conditions. As they used only single-spacecraft measurements they couldn't exclude that these structures were due to the flapping motion of the plasma sheet instead of being filaments detached from the plasma sheet.

Theta auroras are also associated with plasma sheet ion precipitation (e.g. Peterson and Shelley, 1984; Frank et al., 1986). Theta auroras are intense and large scale structures crossing the whole polar cap. However, according to their scale size and because of the low field-aligned potential drops, the PCIB we observe may preferentially correspond to arcs at smaller scale rather than to theta aurora. The presence or lack of precipitating ions has been frequently used to asses if isolated polar cap arcs are located on closed or open field lines. Depending on the study, ion precipitation has been identified (e.g. Ismail et al., 1977; Whalen et al., 1971) or not (e.g. Hoffman et al., 1985; Bonnell et al., 1999). Thus, there still exists some controversy on the issue of whether ion precipitation is associated with polar cap arcs or not, and whether they are located on closed or open field lines. Our study brings supplementary evidence showing that there is no clear difference between PCIB detected together with dense, with tenuous, or with no isotropic ions. The presence or absence of precipitating ions alone shouldn't be used to classify arcs and is not sufficient to know the magnetic field line topology (open or closed). Other data, such as the convection signatures or the properties of precipitating electrons, should also be considered. This should be the topic of future studies aiming to identify the magnetic field lines topology and the source region of the precipitating electrons. Conjugated studies combining Cluster in-situ data with SuperDARN convection maps and/or polar cap images should also be of great interest to shed light on the driver of particle acceleration inside polar cap arcs. 
Acknowledgements. Data analysis was done with the AMDA science analysis system provided by the Centre de Données de la Physique des Plasmas (CESR, Université Paul Sabatier, Toulouse) supported by CNRS and CNES. The authors are grateful to E. Penou for the development of CL, the Cluster CIS software, to the Cluster Active Archive for providing Cluster data, and to the CDAWEB for providing ACE data. R. Maggiolo is supported by a PRODEX/CLUSTER contract (PEA 90096). Marius Echim acknowledges support from the Belgian Office for Science (BELSPO) grant MO/35/031 and from ESA PECS grant 98049/2007.

Topical Editor R. Nakamura thanks two anonymous referees for their help in evaluating this paper.

\section{References}

Balogh, A., Carr, C. M., Acuña, M. H., Dunlop, M. W., Beek, T. J., Brown, P., Fornacon, K.-H., Georgescu, E., Glassmeier, K.H., Harris, J., Musmann, G., Oddy, T., and Schwingenschuh, K.: The Cluster Magnetic Field Investigation: overview of in-flight performance and initial results, Ann. Geophys., 19, 1207-1217, doi:10.5194/angeo-19-1207-2001, 2001.

Bergmann, R., Roth, I., and Hudson, M. K.: Linear stability of the $\mathrm{H}^{+} \mathrm{O}^{+}$two-stream interaction in a magnetized plasma, J. Geophys. Res., 93, 4005-4020, 1988.

Bonnell, J., Elphic, R. C., Palfery, S., Strangeway, R. J., Peterson, W. K., Klumpar, D., Carlson, C. W., Ergun, R. E., and McFadden, J. P.: Observations of polar cap arcs on FAST, J. Geophys. Res., 104, 12669-12682, 1999.

Burke, W. J., Hardy, D. A., Rich, F. J., Gussenhoven, M. S., and Kelley, M. C.: Electric and magnetic field characteristics of discrete arcs in the polar cap, J. Geophys. Res., 87, 2431-2443, 1982.

Chang, T. and Coppi, B.: Lower hybrid acceleration and ion evolution in the suprauroral region, Geophys. Res. Lett., 8, 12531256, 1981.

Davis, T. N.: Negative Correlation between Polar-Cap Visual Aurora and Magnetic Activity, J. Geophys. Res., 68, 4447-4453, 1963.

Décréau, P. M. E., Fergeau, P., Krasnoselskikh, V., Le Guirriec, E., Lévêque, M., Martin, Ph., Randriamboarison, O., Rauch, J. L., Sené, F. X., Séran, H. C., Trotignon, J. G., Canu, P., Cornilleau, N., de Fraudy, H., Alleyne, H., Yearby, K., Mögensen, P. B., Gustafsson, G., André, M., Gurnett, D. C., Darrouzet, F., Lemaire, J., Harvey, C. C., Travnicek, P., and Whisper experimenters (Table 1): Early results from the Whisper instrument on Cluster: an overview, Ann. Geophys., 19, 1241-1258, doi:10.5194/angeo-19-1241-2001, 2001.

De Keyser, J., Maggiolo, R., and Echim, M.: Monopolar and bipolar auroral electric fields and their effects, Ann. Geophys., 28, 2027-2046, doi:10.5194/angeo-28-2027-2010, 2010.

Elphinstone, R. D., Jankowska, K., Murphree, J. S., and Cogger, L. L.: The configuration of the auroral distribution for interplanetary magnetic field Bz northward. I - IMF Bx and By dependencies as observed by the Viking satellite, J. Geophys. Res., 95, 5791-5804, 1990.

Engwall, E., Eriksson, A. I., Cully, C. M., André, M., Puhl-Quinn, P. A., Vaith, H., and Torbert, R.: Survey of cold ionospheric outflows in the magnetotail, Ann. Geophys., 27, 3185-3201, doi:10.5194/angeo-27-3185-2009, 2009.
Ergun, R. E., Carlson, C. W., McFadden, J. P., Mozer, F. S., Delory, G. T., Peria, W., Chaston, C. C., Temerin, M., Elphic, R., Strangeway, R., Pfaff, R., Cattell, C. A., Klumpar, D., Shelley, E., Peterson, W., Moebius, E., and Kistler, L.: FAST satellite observations of electric field structures in the auroral zone, Geophys. Res. Lett., 25, 2025-2028, 1998.

Frank, L. A., Craven, J. D., Gurnett, D. A., Shawhan, S. D., Burch, J. L., Winningham, J. D., Chappell, C. R., Waite, J. H., Maynard, N. C., and Sugiura, M.: The theta aurora, J. Geophys. Res., 91, 3177-3224, 1986.

Fujimoto, M., Nishida, A., Mukai, T., Saito, Y., Yamamoto, T., and Kokubun, S.: Plasma entry from the flanks of the near-Earth magnetotail: Geotail observations in the dawnside LLBL and the plasma sheet, J. Geomagn. Geoelectr., 48, 711-727, 1996.

Grigorenko, E. E., Fedorov, A., and Zelenyi, L. M.: Statistical study of transient plasma structures in magnetotail lobes and plasma sheet boundary layer: Interball-1 observations, Ann. Geophys., 20, 329-340, doi:10.5194/angeo-20-329-2002, 2002.

Gussenhoven, M. S.: Extremely high latitude auroras, J. Geophys. Res., 87, 2401-2412, 1982.

Gustafsson, G., Bostrom, R., Holback, B., Holmgren, G., Lundgren, A., Stasiewicz, K., Ahlen, L., Mozer, F. S., Pankow, D., Harvey, P., Berg, P., Ulrich, R., Pedersen, A., Schmidt, R., Butler, A., Fransen, A. W. C., Klinge, D., Thomsen, M., Falthammar, C.-G., Lindqvist, P.-A., Christenson, S., Holtet, J., Lybekk, B., Sten, T. A., Tanskanen, P., Lappalainen, K., and Wygant, J.: The Electric Field and Wave Experiment for the Cluster Mission, Space Sci. Rev., 79, 137-156, 1997.

Hoffman, R. A., Heelis, R. A., and Prasad, J. S.: A sun-aligned arc observed by DMSP and AE-C, J. Geophys. Res., 90, 9697-9710, 1985.

Ismail, S. and Meng, C.-I.: A classification of polar cap auroral arcs, Planet. Space Sci., 30, 319-330, 1982,

Ismail, S., Wallis, D. D., and Cogger, L. L.: Characteristics of polar cap sun-aligned arcs, J. Geophys. Res., 82, 4741-4749, 1977.

Kletzing, C., Cattell, C., Mozer, F. S., Akasofu, S.-I., and Makita, K.: Evidence for electrostatic shocks as the source of discrete auroral arcs, J. Geophys. Res., 88, 4105-4113, 1983.

Koleva, R. and Sauvaud, J.-A.: Plasmas in the near-Earth magnetotail lobes: Properties and sources, J. Atmos. Terr. Phys., 70, 2118-2131, 2008.

Kullen, A., Brittnacher, M., Cumnock, J. A., and Blomberg, L. G.: Solar wind dependence of the occurrence and motion of polar auroral arcs: A statistical study, J. Geophys. Res., 107, SMP 13-1, 2002.

Lassen, K. and Danielsen, C.: Quiet time pattern of auroral arcs for different directions of the interplanetary magnetic field in the Y-Z plane, J. Geophys. Res., 83, 5277-5284, 1978.

Maggiolo, R., Sauvaud, J. A., Fontaine, D., Teste, A., Grigorenko, E., Balogh, A., Fazakerley, A., Paschmann, G., Delcourt, D., and Rème, H.: A multi-satellite study of accelerated ionospheric ion beams above the polar cap, Ann. Geophys., 24, 1665-1684, doi:10.5194/angeo-24-1665-2006, 2006.

Makita, K., Meng, C.-I., and Akasofu, S.-I.: The Shift of the Auroral Electron Precipitation Boundaries in the Dawn-Dusk Sector in Association with Geomagnetic Activity and Interplanetary Magnetic Field, J. Geophys. Res., 88, 7967-7981, 1983.

Makita, K., Meng, C.-I., and Akasofu, S.-I.: Transpolar auroras, their particle precipitation, and IMF B(y) component, J. Geo- 
phys. Res., 96, 14085-14095, 1991.

Moore, T. E., Chappell, C. R., Chandler, M. O., Craven, P. D., Giles, B. L., Pollock, C. J., Burch, J. L., Young, D. T., Waite Jr., J. H., Nordholt, J. E., Thomsen, M. F., McComas, D. J., Berthelier, J. J., Williamson, W. S., Robson, R., and Mozer, F. S.: Highaltitude observations of the polar wind, Science, 277, 349-351, 1997.

Mozer, F. S. and Hull, A.: Origin and geometry of upward parallel electric fields in the auroral acceleration region, J. Geophys. Res., 106, 5763-5778, 2001.

Mozer, F. S., Carlson, C. W., Hudson, M. K., Torbert, R. B., Parady, B., Yatteau, J., and Kelley, M. C.: Observation of paired electrostatic shocks in the polar magnetosphere, Phys. Rev. Lett., 38, 292-295, 1977

Newell, P. T., Lyons, K. M., and Meng, C.-I.: A large survey of electron acceleration events, J. Geophys. Res., 101, 2599-2614, 1996.

Nilsson, H., Waara, M., Arvelius, S., Marghitu, O., Bouhram, M., Hobara, Y., Yamauchi, M., Lundin, R., Rème, H., Sauvaud, J.A., Dandouras, I., Balogh, A., Kistler, L. M., Klecker, B., Carlson, C. W., Bavassano-Cattaneo, M. B., and Korth, A.: Characteristics of high altitude oxygen ion energization and outflow as observed by Cluster: a statistical study, Ann. Geophys., 24, 1099-1112, doi:10.5194/angeo-24-1099-2006, 2006.

Partamies, N., Donovan, E., and Knudsen, D.: Statistical study of inverted-V structures in FAST data, Ann. Geophys., 26, 14391449, doi:10.5194/angeo-26-1439-2008, 2008.

Paschmann, G., Quinn, J. M., Torbert, R. B., Vaith, H., McIlwain, C. E., Haerendel, G., Bauer, O. H., Bauer, T., Baumjohann, W., Fillius, W., Förster, M., Frey, S., Georgescu, E., Kerr, S. S., Kletzing, C. A., Matsui, H., Puhl-Quinn, P., and Whipple, E. C.: The Electron Drift Instrument on Cluster: overview of first results, Ann. Geophys., 19, 1273-1288, doi:10.5194/angeo-191273-2001, 2001.

Peterson, W. K. and Shelley, E. G.: Origin of the plasma in a crosspolar cap auroral feature (theta aurora), J. Geophys. Res., 89, 6729-6736, 1984.

Rairden, R. L. and Mende, S. B.: Properties of 6300- $\AA$ auroral emissions at South Pole, J. Geophys. Res., 94, 1402-1416, 1989.

Reiff, P. H., Collin, H. L., Craven, J. D., Burch, J. L., and Winningham, J. D.: Determination of auroral electrostatic potentials using high- and low-altitude particle distributions, J. Geophys. Res., 93, 7441-7465, 1988.

Rème, H., Aoustin, C., Bosqued, J. M., Dandouras, I., Lavraud, B., Sauvaud, J. A., Barthe, A., Bouyssou, J., Camus, Th., CoeurJoly, O., Cros, A., Cuvilo, J., Ducay, F., Garbarowitz, Y., Medale, J. L., Penou, E., Perrier, H., Romefort, D., Rouzaud, J., Vallat, C., Alcaydé, D., Jacquey, C., Mazelle, C., d'Uston, C., Möbius, E., Kistler, L. M., Crocker, K., Granoff, M., Mouikis, C., Popecki, M., Vosbury, M., Klecker, B., Hovestadt, D., Kucharek, H., Kuenneth, E., Paschmann, G., Scholer, M., Sckopke, N., Seidenschwang, E., Carlson, C. W., Curtis, D. W., Ingraham, C., Lin, R.
P., McFadden, J. P., Parks, G. K., Phan, T., Formisano, V., Amata, E., Bavassano-Cattaneo, M. B., Baldetti, P., Bruno, R., Chionchio, G., Di Lellis, A., Marcucci, M. F., Pallocchia, G., Korth, A., Daly, P. W., Graeve, B., Rosenbauer, H., Vasyliunas, V., McCarthy, M., Wilber, M., Eliasson, L., Lundin, R., Olsen, S., Shelley, E. G., Fuselier, S., Ghielmetti, A. G., Lennartsson, W., Escoubet, C. P., Balsiger, H., Friedel, R., Cao, J.-B., Kovrazhkin, R. A., Papamastorakis, I., Pellat, R., Scudder, J., and Sonnerup, B.: First multispacecraft ion measurements in and near the Earth's magnetosphere with the identical Cluster ion spectrometry (CIS) experiment, Ann. Geophys., 19, 1303-1354, doi:10.5194/angeo19-1303-2001, 2001.

Rodriguez, J. V., Valladares, C. E., Fukui, K., and Gallagher, H. A.: Antisunward decay of polar cap arcs, J. Geophys. Res., 102, 27227-27248, 1997.

Seki, K., Elphic, R. C., Hirahara, M., Terasawa, T., and Mukai, T.: On Atmospheric Loss of Oxygen Ions from Earth Through Magnetospheric Processes, Science, 291, 1939-1941, 2001.

Terasawa, T., Fujimoto, M., Mukai, T., Shinohara, I., Saito, Y., Yamamoto, T., Machida, S., Kokubun, S., Lazarus, A. J., Steinberg, J. T., and Lepping, R. P.: Solar wind control of density and temperature in the near-Earth plasma sheet: WIND/Geotail collaboration, Geophys. Res. Lett., 24, 935-938, 1997.

Teste, A., Fontaine, D., Sauvaud, J.-A., Maggiolo, R., Canu, P., and Fazakerley, A.: CLUSTER observations of electron outflowing beams carrying downward currents above the polar cap by northward IMF, Ann. Geophys., 25, 953-969, doi:10.5194/angeo-25953-2007, 2007.

Thomsen, M. F., Borovsky, J. E., Skoug, R. M., and Smith, C. W.: Delivery of cold, dense plasma sheet material into the near-Earth region, J. Geophys. Res., 108, SMP 6-1, 1993.

Thomsen, M. F., Borovsky, J. E., Skoug, R. M., and Smith, C. W.: Delivery of cold, dense plasma sheet material into the near-Earth region, J. Geophys. Res., 108, 1151-1159, 2003.

Troshichev, O. A., Gusev, M. G., Nikolashkin, S. V., and Samsonov, V. P.: Features of the polar cap aurorae in the Southern Polar Region, Planet. Space Sci., 36, 429-439, 1988.

Tsyganenko, N. A.: A magnetospheric magnetic field model with a warped tail current sheet, Planet. Space Sci., 37, 5-20, 1989.

Valladares, C. E., Carlson Jr., H. C., and Fukui, K.: Interplanetary magnetic field dependency of stable Sun-aligned polar cap arcs, J. Geophys. Res., 99, 6247-6272, 1994.

Whalen, B. A., Miller, J. R., and McDiarmid, I. B.: Sounding Rocket Observations of Particle Precipitation in a Polar-Cap Electron Aurora, J. Geophys. Res., 76, 6847-6855, 1971.

Wing, S. and Newell, P. T.: Central plasma sheet ion properties as inferred from ionospheric observations, J. Geophys. Res., 103, 6785-6800, 1998.

Yau, A. W., Abe, T., and Peterson, W. K.: The polar wind: Recent observations, J. Atmos. Terrest. Phys., 69, 1936-1983, 2007.

Zhu, L., Schunk, R. W., and Sojka, J. J.: Polar cap arcs: a review, J. Atmos. Terrest. Phys., 59, 1087-1126, 1997. 